\title{
THE DEPLETION OF WATER DURING DISPERSAL OF PLANET-FORMING DISK REGIONS
}

\author{
A. Banzatti ${ }^{1,2}$, K. M. Pontoppidan ${ }^{1}$, C. Salyk $^{3}$, G. J. Herczeg ${ }^{4}$, E. F. van DishoecK ${ }^{5}$, And G. A. Blake ${ }^{6}$ \\ ${ }^{1}$ Space Telescope Science Institute, 3700 San Martin Drive, Baltimore, MD 21218, USA; banzatti@1pl.arizona.edu \\ ${ }^{2}$ Lunar and Planetary Laboratory, The University of Arizona, Tucson, AZ 85721, USA \\ ${ }^{3}$ Vassar College, 124 Raymond Avenue, Poughkeepsie, NY 12604, USA \\ ${ }^{4}$ Kavli Institute for Astronomy and Astrophysics, Peking University, Yi He Yuan Lu 5, Haidian Qu, 100871 Beijing, China \\ ${ }^{5}$ Leiden Observatory, Leiden University, P.O. Box 9513, 2300RA Leiden, The Netherlands \\ ${ }^{6}$ Division of Geological \& Planetary Sciences, MC 150-21, California Institute of Technology, Pasadena, CA 91125, USA \\ Received 2016 September 9; revised 2016 November 6; accepted 2016 November 17; published 2017 January 11
}

\begin{abstract}
We present a new velocity-resolved survey of $2.9 \mu \mathrm{m}$ spectra of hot $\mathrm{H}_{2} \mathrm{O}$ and $\mathrm{OH}$ gas emission from protoplanetary disks, obtained with the Cryogenic Infrared Echelle Spectrometer at the VLT $(R \sim 96,000)$. With the addition of archival Spitzer-IRS spectra, this is the most comprehensive spectral data set of water vapor emission from disks ever assembled. We provide line fluxes at $2.9-33 \mu \mathrm{m}$ that probe from the dust sublimation radius at $\sim 0.05$ au out to the region of the water snow line. With a combined data set for 55 disks, we find a new correlation between $\mathrm{H}_{2} \mathrm{O}$ line fluxes and the radius of $\mathrm{CO}$ gas emission, as measured in velocity-resolved $4.7 \mu \mathrm{m}$ spectra $\left(R_{\mathrm{co}}\right)$, which probes molecular gaps in inner disks. We find that $\mathrm{H}_{2} \mathrm{O}$ emission disappears from $2.9 \mu \mathrm{m}$ (hotter water) to $33 \mu \mathrm{m}$ (colder water) as $R_{\text {co }}$ increases and expands out to the snow line radius. These results suggest that the infrared water spectrum is a tracer of inside-out water depletion within the snow line. It also helps clarify an unsolved discrepancy between water observations and models by finding that disks around stars of $M_{\star}>1.5 M_{\odot}$ generally have inner gaps with depleted molecular gas content. We measure radial trends in $\mathrm{H}_{2} \mathrm{O}, \mathrm{OH}$, and $\mathrm{CO}$ line fluxes that can be used as benchmarks for models to study the chemical composition and evolution of planet-forming disk regions at 0.05-20 au. We propose that JWST spectroscopy of molecular gas may be used as a probe of inner disk gas depletion, complementary to the larger gaps and holes detected by direct imaging and by ALMA.
\end{abstract}

Key words: circumstellar matter - molecular processes - planets and satellites: formation - protoplanetary disks stars: pre-main sequence

\section{INTRODUCTION}

Protoplanetary disks are composed of the circumstellar material that feeds stars and planets. These systems evolve from early gas- and dust-rich phases to phases of dispersal, eventually unveiling planetary systems that form within them (e.g., Alexander et al. 2014, p. 475). Evidence is growing that there is at least one mode of disk dispersal, working from the inside out. Observations of a wavelength-dependent decrease in infrared excesses (e.g., Skrutskie et al. 1990; Haisch et al. 2001; Espaillat et al. 2012; Ribas et al. 2015), or spatially resolved cavities in continuum dust emission (e.g., Andrews et al. 2011; van der Marel et al. 2016), indicate that dust grains in the inner disk regions are depleted before those at larger radii. Disks that show these properties have been proposed to be in a transition phase ("transitional disks") toward their dispersal (e.g., Strom et al. 1989; Skrutskie et al. 1990; Takeuchi \& Artymowicz 2001). The concept of disks in "transition" has been developing ever since its early definition, under new evidence provided by larger samples, more detailed observations, and improved models (e.g., Espaillat et al. 2014; Owen 2016). Understanding the properties, origin, and evolution of inner disk dispersal and of gaps in protoplanetary disks bears the potential to indicate when, where, and in what physical and chemical environments planets form.

Models that produce an inside-out dispersal of disks include the effects of photoevaporation by stellar X-ray and UV radiation (e.g., Alexander et al. 2006; Gorti \& Hollenbach 2009; Owen et al. 2010). In these models, disk gaps are opened where thermal heating launches the gas into a photoevaporative wind by providing enough energy to exceed the gravitational potential of the star. After a gap is opened, the inner disk is expected to be drained onto the central star by viscous accretion, and the inner hole would rapidly grow to larger radii under the effect of photoevaporation. Disks may also disperse under removal of gas by magnetohydrodynamic (MHD) winds (e.g., Ferreira et al. 2006; Bai 2016). Another process that has been shown to open gaps in disks is the formation of giant planets (e.g., Lin \& Papaloizou 1986; Kley \& Nelson 2012, among several others). Where and when gaps may be opened depends on the efficiency of giant planet core formation (usually assumed to increase close to the water snow line, at several to $\sim 10 \mathrm{au}$; e.g., Ida \& Lin 2005; Kennedy \& Kenyon 2008), on their migration, and on the number of planets (e.g., Zhu et al. 2011; Dong et al. 2015). In this scenario the inner region within the gap opened by the planet will also be depleted by viscous accretion onto the central star, eventually forming an inner disk hole. While there is no single model that can entirely match the currently observed properties of "transitional" disks, there is evidence that the photoevaporative and planet scenarios are both happening in disks (Owen 2016). Models are now also exploring the combination of multiple processes, toward a more comprehensive understanding of the evolution of planet-forming disk regions. Recent models by Gorti et al. (2015), for instance, explore the combination of dust evolution and disk photoevaporation, showing how the gas-to-dust ratio decreases in photoevaporating inner disks at radii of $\lesssim 10 \mathrm{au}$, producing favorable conditions for rocky planetesimal formation.

The detailed study of the properties of inner disk gaps and holes is greatly advancing thanks to the increase in spatial and 
Table 1

Summary of Major Water Vapor Surveys in Protoplanetary Disks

\begin{tabular}{|c|c|c|c|c|c|c|c|c|c|}
\hline $\begin{array}{l}\lambda \\
(\mu \mathrm{m})\end{array}$ & Instr. & Resol. & \# Lines & $\begin{array}{c}E_{u} \\
(\mathrm{~K})\end{array}$ & \# Disks & $M_{\star}<0.2$ & $\begin{array}{l}0.2<M_{\star}<1.5 \\
\text { on Fractions (and s }\end{array}$ & $\begin{array}{l}\qquad M_{\star}>1.5 \\
\text { nple sizes) }\end{array}$ & Refs \\
\hline \multirow[t]{2}{*}{$2.9-3$} & VLT- & 100,000 & $\approx 40$ & $8000-10,000$ & $900 \approx 40$ & $\mathrm{~N} / \mathrm{A}$ & $58 \%(24)$ & $11 \%(18)$ & this work, (1) \\
\hline & CRIRES & & & & Sensitivity: & $\ldots$ & -15 & -14.6 & \\
\hline \multirow[t]{2}{*}{$10-37$} & Spitzer- & 700 & $\approx 200$ & $700-6000$ & $300-700 \approx 100$ & $0 \%(5)$ & $63 \%-85 \%(64)$ & $0 \%-18 \%$ (27) & this work, (2) \\
\hline & IRS & & & & Sensitivity: & -15.7 & -14.8 & -13.7 & \\
\hline \multirow[t]{2}{*}{$55-200$} & Herschel- & $1000-5000$ & $\approx 20$ & $100-1400$ & $100-300 \approx 120$ & $0 \%(10)$ & $15 \%(80)$ & $15 \%(27)$ & (3) \\
\hline & PACS & & & & Sensitivity: & -14.6 & -14.5 & -14 & \\
\hline
\end{tabular}

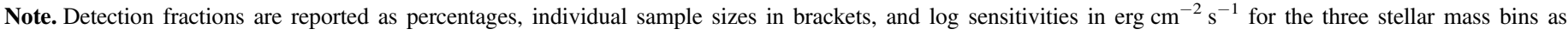
indicated.

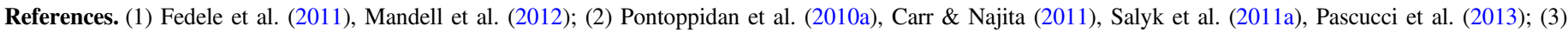
Riviere-Marichalar et al. (2012, 2015), Meeus et al. (2012), Fedele et al. (2013), Blevins et al. (2016).

spectral resolution of observing techniques. Direct imaging is able to reveal gap sizes (if their angular size is large enough to be spatially resolved) and the relative distribution of dust grain populations (small versus large grains; Garufi et al. 2013; Akiyama et al. 2016), providing tracers of the physical processes that act in inner disks. Gas spectroscopy holds a unique potential to unveil the chemical evolution of planetforming regions. Recent developments in sensitivity and spectral resolution of infrared spectrographs allowed the first large surveys of molecular gas in inner disks able to spectrally (and sometimes also spatially) resolve gas within dust gaps (e.g., Pontoppidan et al. 2008; Salyk et al. 2011a; van der Plas et al. 2015). The combined analysis of a large sample of disks recently established rovibrational $\mathrm{CO}$ (carbon monoxide) emission at $4.7 \mu \mathrm{m}$ as a tracer of inner gaps in molecular gas. Banzatti \& Pontoppidan (2015) found evidence for an insideout depletion sequence where $\mathrm{CO}$ gas is progressively removed from $\sim 0.1$ out to $\sim 20 \mathrm{au}$, providing a new probe of gaps over a radial disk region that extends the region probed by millimeter interferometers (typically limited to $\gtrsim 5-10 \mathrm{au}$, depending on the distance from Earth). By tracing $\mathrm{H}_{2}$ emission at ultraviolet wavelengths, Hoadley et al. (2015) found similar evidence for a removal of molecular gas at small radii in transitional disks. The combination of dust and gas studies of small to large gaps bears the potential for a comprehensive understanding of the physical and chemical evolution of inner disks during planet formation. As a further contribution to this rapidly growing field, we report on the depletion of $\mathrm{H}_{2} \mathrm{O}$ (water) and $\mathrm{OH}$ (hydroxyl) during the formation of inner disk gaps.

\subsection{An Unsolved Mystery from Water Surveys: Low Detections in Disks Around Intermediate-mass Stars}

Water vapor emission has been observed in protoplanetary disks between 3 and $540 \mu \mathrm{m}$, with significant differences in detection rates, depending on the observed wavelength and on the mass of the central star. The most comprehensive survey of water emission in disks by a single instrument has been obtained by the IRS spectrograph on the Spitzer Space Telescope (Houck et al. 2004; Werner et al. 2004), which measured hundreds of water emission lines between 10 and $37 \mu \mathrm{m}$ in over 60 disks. Spitzer observations showed that water emission is strongly dependent on the stellar spectral type and tentatively dependent on the evolutionary stage, with high detection rates in disks around solar-mass stars, low detection rates in disks around intermediate-mass stars, and similarly low rates in a small sample of "transitional" disks $(1 / 6$; see Table 1 and Pontoppidan et al. 2010a; Carr \& Najita 2011; Salyk et al. 2015). Water emission at Spitzer wavelengths has been interpreted in most disks as coming from an optically thick surface layer within the snow line radius, at temperatures of 300-700 K (Carr \& Najita 2011; Salyk et al. 2011a). Modeling of the velocity unresolved Spitzer spectra $\left(\Delta \mathrm{v} \sim 400 \mathrm{~km} \mathrm{~s}^{-1}\right)$ provided estimates of the disk emitting region to the inner few au in disks (e.g., Najita et al. 2011; Antonellini et al. 2015; Walsh et al. 2015). At $12.4 \mu \mathrm{m}$, within the Spitzer-IRS range, a few water lines have been resolved in velocity in four disks with VLT-VISIR and Gemini-TEXES $(\Delta \mathrm{v} \sim 15$ and $\sim 3.5 \mathrm{~km} \mathrm{~s}^{-1}$, respectively; Lagage et al. 2004; Lacy et al. 2002), providing support to the emitting regions estimated by models for the Spitzer spectra (Pontoppidan et al. 2010b; Banzatti et al. 2014; Salyk et al. 2015). At shorter wavelengths $(\sim 2-3 \mu \mathrm{m})$, hotter water $(T \approx 900-1500 \mathrm{~K})$ has been spectrally resolved and analyzed in only four disks so far, by Salyk et al. (2008) and Mandell et al. (2012) with Keck-NIRSPEC and VLT-CRIRES, and by Carr et al. (2004) with IRTF-CSHELL. At longer wavelengths $(>55 \mu \mathrm{m})$, colder water has been surveyed in disks by Herschel-PACS, finding similarly low detection rates in disks around solar-mass as in disks around intermediate-mass stars $(\sim 15 \%$ as based on the $63.3 \mu \mathrm{m}$ line; see references in Table 1 and Rivière Marichalar et al. 2016).

Table 1 summarizes the properties, samples, and detection rates for all major surveys of water vapor in protoplanetary disks, including this work. The general trends identified to date are shown in Figure 1, and are compared to expectations produced by recent models of water in disks (Antonellini et al. 2015; Walsh et al. 2015). ${ }^{7}$ We investigate the measured line fluxes against the masses of the central stars, $M_{\star}$, as an alternative way to look at the spectral-type dependencies found by Pontoppidan et al. (2010a). Water line fluxes generally increase with stellar luminosity (Salyk et al. 2011a), and with stellar mass. Models explain this with the radial extent of the warm $(>300 \mathrm{~K})$ disk surface, where water vapor is abundant due to ice evaporation and efficient gas-phase formation, which increases to larger disk radii around more luminous stars. Model predictions agree well with the increase in Spitzer line fluxes measured in disks around stars of masses between 0.2 and $1.5 M_{\odot}$, although $20 \%-40 \%$ of disks show significantly

\footnotetext{
7 We reproduce the model spectra of Walsh et al. (2015) by using a slab model in LTE and adopting the temperature, column density, and emitting area reported for the observable layer of gas (down to $\tau=1$ ); in the case of Antonellini et al. (2016), we use the model spectra kindly provided by the authors. We scale the models to the line fluxes measured in disks around stars of $0.5 M_{\odot}$, to highlight global trends with $M_{\star}$.
} 


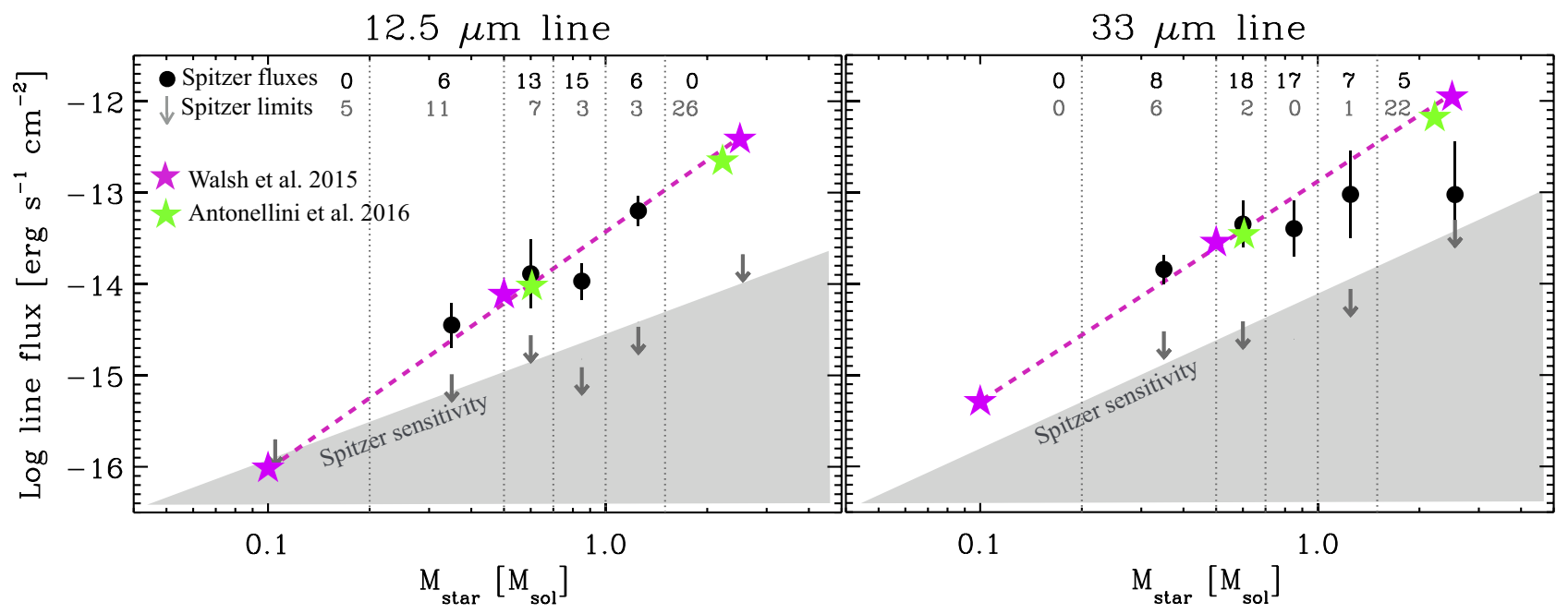

Figure 1. Mid-IR water emission dependence on stellar mass. Black dots and error bars show median values and the median absolute deviation of water line fluxes measured in Spitzer-IRS spectra. Median $1 \sigma$ flux upper limits are shown with gray arrows. The number of disks where water emission is detected/undetected in each stellar mass bin is shown at the top. Models by Walsh et al. (2015) and Antonellini et al. (2016) are scaled to the measured line fluxes in disks around stars of $0.5 M_{\odot}$. All fluxes are normalized to a common distance of $140 \mathrm{pc}$.

lower fluxes relative to what is expected from a pure luminosity effect. For $M_{\star}>1.5 M_{\odot}$, models produce water line fluxes $\gtrsim 10$ times stronger than observed for $80 \%-100 \%$ of the disk sample. The low water detection frequency at infrared wavelengths in disks around stars of $M_{\star}>1.5 M_{\odot}$ is still not understood (e.g., Walsh et al. 2015).

In this work, we test the hypothesis that inside-out inner disk depletion may produce the progressive disappearance of water vapor emission at infrared wavelengths. If water vapor in inner disks is depleted from smaller to larger disk radii, an essential spectral region to include in the investigation of this process is where the hot water emits, in high-energy transitions at 2.9-3 $\mathrm{m}$ (e.g., Mandell et al. 2012). In Sections 2 and 3 we describe the new survey of hot water emission as observed with CRIRES at $2.9 \mu \mathrm{m}$, and the comparative analysis with the CO data set at $4.7 \mu \mathrm{m}$. In Section 4 we report the discovery of a correlation between water line fluxes at $2.9-33 \mu \mathrm{m}$ and the size of a gas-depleted region in the inner disk, as probed by rovibrational $\mathrm{CO}$ emission. We discuss the properties of these molecular gaps, and propose their potential for studies of the evolution of planet-forming disk regions, in Section 5.

\subsection{A Note on Molecular Gaps/Holes in Inner Disks}

The terms disk "gap" and "hole" have referred (sometimes interchangeably) to radial regions where the dust in the disk shows some degree of depletion, as compared to a continuous distribution adopted by models for disks considered "primordial" (e.g., Espaillat et al. 2012). In this work, we adopt these terms to identify inner disk regions of various sizes (namely $\approx 0.1-20 \mathrm{au}$ ), empirically defined to where molecular gas is depleted enough that its infrared emission (at wavelengths of $2.9-35 \mu \mathrm{m}$ ) is not detected. The term "gap" was utilized in Banzatti \& Pontoppidan (2015) with this meaning, with the discovery of a strong correlation between velocity-resolved line widths and the $v 2 / v 1$ vibrational ratios in rovibrational CO emission at $4.7 \mu \mathrm{m}$ in a large survey of protoplanetary disks. By converting these measurements respectively into a characteristic $\mathrm{CO}$ emitting radius $R_{\mathrm{co}}$ and the vibrational excitation temperature, this correlation revealed cooler $\mathrm{CO}$ gas as larger gaps are formed in disks (i.e., as $R_{\text {co }}$ increases). This observational finding suggests an inside-out depletion scenario, which is now being increasingly supported by independent modeling explorations (Hein Bertelsen et al. 2016; Woitke et al. 2016). To distinguish them from dust gaps or holes detected by other techniques, we call them "molecular gaps/ holes," especially after the discovery in this work that they are shared by three of the most abundant molecules in disks: $\mathrm{CO}$, $\mathrm{H}_{2} \mathrm{O}$, and $\mathrm{OH}$ (Sections 3 and 4). In the absence of constraints on the radial distribution of any undetected residual $\mathrm{CO}$ gas within the CO-depleted inner region, we use the terms "gap" and "hole" interchangeably. An upper limit to the CO column density of $\lesssim 10^{15} \mathrm{~cm}^{-2}$ has been estimated by Carmona et al. (2016) in one of these gaps/holes, implying that very little residual $\mathrm{CO}$ gas is left (if any) in the inner CO-depleted region.

\section{OBSERVATIONS AND SAMPLE}

$\mathrm{CO}$ emission at $4.7 \mu \mathrm{m}$ has been observed with the Cryogenic Infrared Echelle Spectrometer (CRIRES; Kaeufl et al. 2004) on the Very Large Telescope (VLT) of the European Southern Observatory (ESO). Most spectra have been taken as part of the ESO Large Program 179.C-0151 (Pontoppidan et al. 2011b); some measurements have been added from programs 079.C-0349 and 081.C-0833 as published in van der Plas et al. (2015), from program 091.C-0671 as published in Carmona et al. (2016), and from program 088. C-0898 as published in Hein Bertelsen et al. (2016). CRIRES has a resolving power of $\sim 96,000$ or $3.2 \mathrm{~km} \mathrm{~s}^{-1}$, which provides detailed velocity-resolved line profiles of $\mathrm{CO}$ gas from inner disks and allows us to radially locate the emission through Keplerian line broadening (e.g., Brown et al. 2013; Banzatti \& Pontoppidan 2015; van der Plas et al. 2015). In total, we include in this work 52 disks that have velocityresolved CO lines from CRIRES observations.

Within the ESO Large Program 179.C-0151, 28 disks were observed at $2.9 \mu \mathrm{m}$ to measure their $\mathrm{H}_{2} \mathrm{O}$ and $\mathrm{OH}$ emission spectra. Three of these disks were previously published in Mandell et al. (2012). Three main-sequence stars were observed to be used as photospheric standards, sampling stellar spectral types from $\mathrm{K}$ to $\mathrm{M}$ (with spectral types taken from Gray et al. 2006; Koen et al. 2010): NLTT29176 (K3), 
Table 2

Sample Properties Adopted or Calculated from the Literature

\begin{tabular}{|c|c|c|c|c|c|c|c|c|c|c|c|c|}
\hline Name & $\begin{array}{c}M_{\star} \\
\left(M_{\odot}\right)\end{array}$ & $\begin{array}{c}L_{\star} \\
\left(L_{\odot}\right)\end{array}$ & $\begin{array}{l}\log M_{\mathrm{acc}} \\
\left(M_{\odot} \mathrm{yr}^{-1}\right)\end{array}$ & $\begin{array}{c}\log L_{\mathrm{acc}} \\
\left(L_{\odot}\right)\end{array}$ & $\begin{array}{c}\text { incl. } \\
\left({ }^{\circ}\right)\end{array}$ & $v 2 / v 1$ & $\begin{array}{l}R_{\mathrm{co}} \\
(\mathrm{au})\end{array}$ & $\begin{array}{c}R_{\text {snow }} \\
(\mathrm{au})\end{array}$ & $\begin{array}{l}\text { Dist. } \\
(\mathrm{pc})\end{array}$ & $\begin{array}{c}\text { Cont. }(3.3 \mu \mathrm{m}) \\
\text { (Jy) }\end{array}$ & $\begin{array}{c}\text { Cont. }(4.6 \mu \mathrm{m}) \\
(\mathrm{Jy})\end{array}$ & References \\
\hline AATau & 0.85 & 0.71 & -8.31 & -1.43 & 71 & 0.14 & 0.20 & 1.45 & 140 & $0.322 \pm 0.011$ & $0.337 \pm 0.006$ & \\
\hline AS205 N & 1.10 & 7.10 & -7.10 & -0.18 & 20 & 0.38 & 0.17 & 5.45 & 125 & $4.390 \pm 0.370$ & $5.220 \pm 0.400$ & \\
\hline AS209 & 1.40 & 2.50 & -7.52 & -0.35 & 39 & 0.91 & 0.08 & 3.84 & 125 & $0.829 \pm 0.055$ & $0.806 \pm 0.032$ & \\
\hline CVCha & 2.10 & 8.00 & -6.81 & 0.41 & 30 & 0.25 & 0.18 & 9.09 & 215 & $0.816 \pm 0.020$ & $1.170 \pm 0.030$ & 1 \\
\hline CWTau & 1.20 & 0.76 & -7.80 & -0.49 & 28 & 0.32 & 0.32 & 2.74 & 140 & $1.470 \pm 0.180$ & $1.760 \pm 0.160$ & \\
\hline DFTau & 0.53 & 1.97 & -6.93 & -0.63 & 65 & 0.43 & 0.20 & 5.08 & 140 & $1.190 \pm 0.130$ & $1.380 \pm 0.090$ & \\
\hline DGTau & 0.30 & 1.70 & -6.39 & 0.70 & 18 & 0.59 & 0.01 & 7.30 & 140 & $1.030 \pm 0.070$ & $1.500 \pm 0.080$ & 2 \\
\hline DoAr24E S & 0.70 & 8.80 & -7.44 & -0.92 & 20 & 0.30 & 0.04 & 3.31 & 120 & $2.360 \pm 0.380$ & $3.130 \pm 0.340$ & \\
\hline DoAr44 & 1.40 & 1.40 & -8.43 & -1.16 & 25 & 0.03 & 0.33 & 1.51 & 125 & $0.610 \pm 0.031$ & $0.593 \pm 0.014$ & \\
\hline DOTau & 0.72 & 1.05 & -7.28 & -0.67 & 31 & 0.49 & 0.12 & 3.93 & 140 & $0.892 \pm 0.080$ & $1.150 \pm 0.060$ & \\
\hline DRTau & 1.00 & 0.90 & -6.82 & -0.24 & 9 & 0.39 & 0.06 & 7.03 & 140 & $1.420 \pm 0.150$ & $1.910 \pm 0.160$ & \\
\hline EC82 & 0.75 & 3.21 & $\cdots$ & 0.03 & 59 & 0.03 & 0.81 & $\cdots$ & 415 & $0.348 \pm 0.008$ & $0.498 \pm 0.010$ & 3 \\
\hline EXLup08 & 0.80 & 0.60 & -7.50 & 0.30 & 44 & 0.61 & 0.06 & 3.25 & 155 & $1.300 \pm 0.300$ & $4.200 \pm 0.600$ & 4 \\
\hline EXLup14 & 0.80 & 0.60 & -10.00 & -1.30 & 48 & 0.51 & 0.07 & 0.25 & 155 & $0.290 \pm 0.040$ & $0.340 \pm 0.030$ & 5 \\
\hline FNTau & 0.33 & 0.80 & -8.25 & -1.77 & 20 & $<0.12$ & 1.69 & 1.12 & 140 & $0.294 \pm 0.009$ & $0.308 \pm 0.005$ & 6 \\
\hline FZTau & 0.70 & 0.51 & -7.33 & -0.27 & 38 & 0.20 & 0.98 & 3.70 & 140 & $1.060 \pm 0.070$ & $1.340 \pm 0.070$ & \\
\hline GQLup & 0.80 & 0.80 & -8.15 & -1.10 & 65 & 0.28 & 0.20 & 1.67 & 150 & $1.090 \pm 0.090$ & $1.030 \pm 0.050$ & \\
\hline HD36112 & 2.00 & 30.00 & -7.35 & -0.10 & 22 & 0.03 & 2.26 & 5.15 & 200 & $4.100 \pm 0.320$ & $4.850 \pm 0.390$ & \\
\hline HD95881 & 2.00 & 2.98 & $<-5.65$ & $<1.63$ & 55 & 0.06 & 4.65 & $<29.31$ & 170 & $4.830 \pm 0.900$ & $6.990 \pm 0.970$ & \\
\hline HD97048 & 2.20 & 33.00 & $<-8.16$ & $<-0.55$ & 43 & 0.33 & 12.57 & $<2.32$ & 180 & $3.010 \pm 0.200$ & $3.250 \pm 0.130$ & \\
\hline HD98922 & 2.20 & 891.00 & $<-6.97$ & $<0.41$ & 45 & 0.29 & 8.85 & $<7.84$ & 350 & $16.500 \pm 2.400$ & $30.700 \pm 0.400$ & \\
\hline HD101412 & 2.30 & 1.36 & $<-7.61$ & $<-0.04$ & 80 & $\ldots$ & 0.79 & $<4.13$ & 160 & $1.090 \pm 0.060$ & $1.720 \pm 0.090$ & \\
\hline HD135344B & 1.60 & 8.00 & -8.35 & -1.11 & 14 & 0.05 & 1.70 & 1.72 & 140 & $3.170 \pm 0.390$ & $3.540 \pm 0.400$ & \\
\hline HD139614 & 1.50 & 6.60 & -7.63 & -0.10 & 20 & 0.25 & 2.77 & 3.51 & 140 & $1.370 \pm 0.070$ & $1.560 \pm 0.040$ & \\
\hline HD141569 & 1.90 & 19.10 & -7.65 & -0.05 & 53 & 0.56 & 17.02 & 3.72 & 100 & $0.652 \pm 0.026$ & $0.441 \pm 0.008$ & \\
\hline HD142527 & 3.50 & 69.00 & -7.02 & 0.07 & 20 & 0.04 & 2.33 & 8.69 & 140 & $6.090 \pm 0.510$ & $7.850 \pm 0.500$ & \\
\hline HD144432S & 1.70 & 14.80 & -7.69 & -0.35 & 27 & 0.09 & 0.78 & 3.44 & 160 & $2.200 \pm 0.360$ & $2.530 \pm 0.260$ & \\
\hline HD150193 & 1.90 & 16.20 & -7.45 & 0.10 & 38 & 0.24 & 0.88 & 4.57 & 150 & $5.130 \pm 1.260$ & $6.470 \pm 1.220$ & \\
\hline HD163296 & 2.30 & 36.00 & -7.13 & 0.28 & 46 & 0.14 & 1.40 & 6.75 & 122 & $9.810 \pm 2.630$ & $11.600 \pm 3.600$ & \\
\hline HD179218 & 2.70 & 75.90 & -6.76 & 0.42 & 57 & 0.37 & 20.80 & 10.40 & 240 & $3.550 \pm 0.700$ & $4.820 \pm 0.590$ & \\
\hline HD190073 & 2.85 & 83.20 & -5.82 & 1.38 & 23 & 0.27 & 6.03 & 27.72 & 767 & $3.950 \pm 0.790$ & $5.620 \pm 1.170$ & \\
\hline HD244604 & 3.05 & 97.70 & -7.19 & 0.14 & 50 & 0.11 & 2.76 & 6.98 & 336 & $0.742 \pm 0.025$ & $0.840 \pm 0.019$ & \\
\hline HD250550 & 3.40 & 190.00 & -5.63 & 1.82 & 10 & 0.16 & 0.91 & 35.70 & 280 & $1.690 \pm 0.210$ & $2.030 \pm 0.160$ & \\
\hline HTLup & 2.50 & 14.50 & $\ldots$ & -0.71 & 28 & 0.65 & 0.05 & $\ldots$ & 150 & $1.500 \pm 0.170$ & $1.590 \pm 0.110$ & \\
\hline IMLup & 0.52 & 1.30 & -11.00 & -1.08 & 49 & 1.16 & 0.03 & 0.08 & 190 & $0.500 \pm 0.023$ & $0.423 \pm 0.009$ & \\
\hline IRS48 & 2.00 & 14.30 & -8.40 & -0.89 & 42 & 0.38 & 16.22 & 1.76 & 125 & $1.490 \pm 0.140$ & $2.220 \pm 0.230$ & \\
\hline LkHa330 & 2.50 & 16.00 & -7.66 & -0.46 & 12 & 0.09 & 4.74 & 4.04 & 250 & $1.130 \pm 0.110$ & $1.360 \pm 0.090$ & \\
\hline RNO90 & 1.50 & 5.70 & -7.40 & -0.19 & 37 & 0.21 & 0.23 & 4.44 & 125 & $2.070 \pm 0.130$ & $2.460 \pm 0.160$ & \\
\hline RULup & 0.70 & 0.42 & -7.75 & -0.47 & 35 & 0.37 & 0.14 & 2.41 & 150 & $1.220 \pm 0.140$ & $1.700 \pm 0.110$ & \\
\hline RYLup & 1.50 & 2.60 & -7.50 & 0.08 & 68 & $<0.04$ & 14.13 & 4.01 & 150 & $1.020 \pm 0.080$ & $1.340 \pm 0.050$ & 7 \\
\hline RWAur & 1.34 & 1.70 & -7.50 & -0.20 & 55 & 0.62 & 0.05 & 3.86 & 140 & $1.000 \pm 0.040$ & $1.380 \pm 0.040$ & 8 \\
\hline SCrA S & 0.60 & 0.76 & -7.53 & -0.65 & 26 & 0.40 & 0.04 & 2.87 & 130 & $2.710 \pm 0.310$ & $3.040 \pm 0.220$ & \\
\hline SCrA N & 1.50 & 2.30 & -7.36 & -0.13 & 10 & 0.30 & 0.05 & 4.63 & 130 & $2.710 \pm 0.310$ & $3.040 \pm 0.220$ & \\
\hline SR9 & 1.35 & 1.41 & -9.20 & $\ldots$ & 60 & $<0.07$ & 0.47 & 0.68 & 120 & $0.729 \pm 0.049$ & $0.660 \pm 0.018$ & 9 \\
\hline SR21 & 2.20 & 15.00 & -7.90 & -0.70 & 15 & 0.64 & 6.46 & 3.03 & 125 & $1.110 \pm 0.090$ & $1.080 \pm 0.040$ & 10 \\
\hline TTau N & 2.10 & 7.30 & -7.16 & 0.05 & 20 & 0.57 & 0.07 & 6.35 & 147 & $8.800 \pm 0.800$ & $12.000 \pm 0.800$ & \\
\hline TTau S & 2.50 & $\cdots$ & $\cdots$ & 0.55 & 25 & 0.20 & 1.10 & $\cdots$ & 147 & $8.800 \pm 0.800$ & $12.000 \pm 0.800$ & \\
\hline TWCha & 1.00 & 0.38 & -8.13 & -1.66 & 67 & 0.16 & 0.71 & 1.84 & 180 & $0.178 \pm 0.004$ & $0.174 \pm 0.003$ & 6 \\
\hline TWHya & 0.70 & 0.23 & -8.70 & -1.30 & 4 & 0.09 & 0.34 & 0.91 & 54 & $0.482 \pm 0.023$ & $0.303 \pm 0.006$ & \\
\hline VSSG1 & 0.52 & 1.50 & -7.56 & -0.87 & 53 & 0.36 & 0.27 & 2.65 & 120 & $0.757 \pm 0.045$ & $1.010 \pm 0.060$ & \\
\hline VVSer & 3.00 & 85.00 & -7.13 & 0.33 & 65 & 0.16 & 2.89 & 7.38 & 415 & $2.600 \pm 0.480$ & $2.530 \pm 0.180$ & \\
\hline VWCha & 0.60 & 3.37 & -7.50 & -0.77 & 44 & 0.32 & 0.16 & 2.95 & 180 & $1.060 \pm 0.050$ & $0.847 \pm 0.047$ & \\
\hline VZCha & 0.80 & 0.46 & -7.91 & -0.73 & 25 & 0.44 & 0.18 & 2.14 & 180 & $0.354 \pm 0.009$ & $0.397 \pm 0.007$ & \\
\hline WaOph6 & 0.90 & 0.67 & -6.64 & -0.73 & 39 & 0.33 & 0.07 & 8.15 & 120 & $1.010 \pm 0.070$ & $1.000 \pm 0.050$ & \\
\hline WXCha & 0.60 & 0.77 & -8.47 & $\ldots$ & 87 & 0.45 & 0.11 & 1.09 & 180 & $0.399 \pm 0.011$ & $0.392 \pm 0.007$ & 11 \\
\hline
\end{tabular}

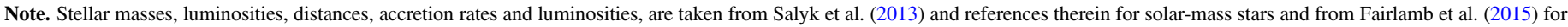

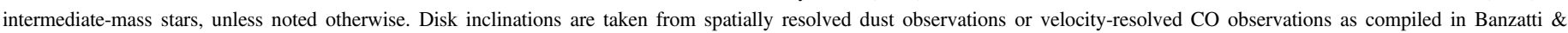

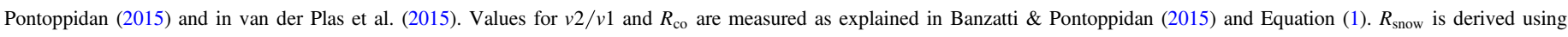

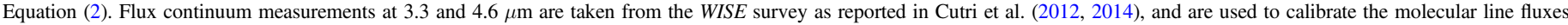

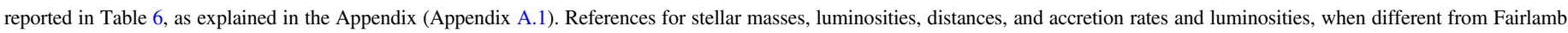

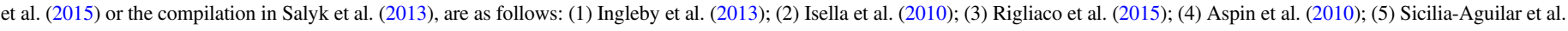

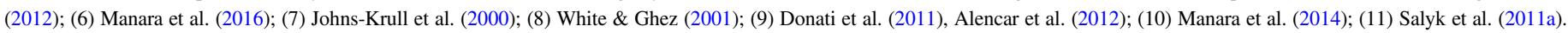

HD111631 (K7), and HIP49986 (M1.5). These spectra are used to correct the $2.9 \mu \mathrm{m} \mathrm{H}_{2} \mathrm{O}$ and $\mathrm{OH}$ emission spectra, as explained in Section 3.1. We also include a second epoch of the 2.9 and $4.7 \mu \mathrm{m}$ spectra of a strongly variable accreting source,
EX Lupi, obtained as part of program 093.C-0432 (Banzatti et al. 2015), and measurements at $2.9 \mu \mathrm{m}$ for HD 250550 and HD 98922 from program 082.C-0491 as published in Fedele et al. (2011). The CRIRES data from programs 179.C-0151 and 
093.C-0432 have been reduced using procedures developed for the ESO Large Program 179.C-0151 as described in Pontoppidan et al. (2011a). To complement the CRIRES data set, we include in this work the Keck-NIRSPEC $(R \sim 25,000$; McLean et al. 1998) $4.7 \mu \mathrm{m}$ CO spectra of DO Tau, HD 244604, and HD 36112, previously published in Blake \& Boogert (2004) and Salyk et al. (2011b). These targets have $\mathrm{H}_{2} \mathrm{O}$ spectra available at $2.9 \mu \mathrm{m}$ and/or at $10-35 \mu \mathrm{m}$, but have not been observed at $4.7 \mu \mathrm{m}$ with CRIRES.

The data set of $\mathrm{H}_{2} \mathrm{O}$ and $\mathrm{CO}$ emission at 2.9 and $4.7 \mu \mathrm{m}$ is analyzed in this work in combination with Spitzer-IRS spectra covering $\mathrm{H}_{2} \mathrm{O}$ emission at $10-35 \mu \mathrm{m}$ at the resolution of $R \sim$ 700. The Spitzer data set has been presented and analyzed in previous work (Pontoppidan et al. 2010a; Carr \& Najita 2011; Salyk et al. 2011a). The advantage of a combined analysis of water emission over such a large wavelength range is to include lines that probe upper level energies between 1500 and $9000 \mathrm{~K}$ and the whole disk region out to the water snow line (see references in Section 1.1). The combined sample includes 55 disks around stars with estimated masses between 0.3 and 3.5 $M_{\odot}$ (Table 2), observed in different star-forming regions (mainly Taurus, Ophiucus, Lupus, Chamaeleon for solar-mass stars, and several isolated intermediate-mass stars), and spanning evolutionary phases from primordial to transitional disks. The sample has been assembled from previous work as driven by the availability of $\mathrm{CO}$ spectra at $4.7 \mu \mathrm{m}$ (available for all 55 disks) and $\mathrm{H}_{2} \mathrm{O}$ spectra at $2.9 \mu \mathrm{m}$ (available for 31 disks in this sample) and/or at 10-35 $\mu \mathrm{m}$ (available for 44 disks in this sample).

\section{SPECTRAL LINE ANALYSIS}

In this section, we present the analysis of the $\mathrm{H}_{2} \mathrm{O}$ and $\mathrm{OH}$ emission spectra in the CRIRES setting at 2.905-2.99 $\mu \mathrm{m}$. The analysis of these spectra presents two main challenges: one due to stellar photosphere absorption and one due to the blending of emission lines, which can both be addressed by exploiting the high spectral resolution provided by CRIRES. The level of photospheric absorption at $2.9 \mu \mathrm{m}$ depends on the spectral type of the star (e.g., a cooler M1 star has more photospheric absorption from $\mathrm{H}_{2} \mathrm{O}$ and $\mathrm{OH}$ than a hotter $\mathrm{K} 3$ star; see Figure 2) and on the amount of veiling from hot dust emission (the ratio between continuum and photospheric flux, $\left.F_{\text {cont }} / F_{\text {phot }}\right)$. We use photospheric templates to correct for photospheric absorption and retrieve the signal from disk gas emission (Section 3.1). Most of the disk emission lines are blended due to their large velocity widths produced by Keplerian rotation in the inner disk (with full widths at half maximum FWHM up to $200 \mathrm{~km} \mathrm{~s}^{-1}$ ). We use the velocityresolved $\mathrm{CO}$ line profiles observed at $4.7 \mu \mathrm{m}$ to characterize the $\mathrm{H}_{2} \mathrm{O}$ and $\mathrm{OH}$ emission at $2.9 \mu \mathrm{m}$ (Section 3.3). The reason to use $\mathrm{CO}$ emission at $4.7 \mu \mathrm{m}$ is that it includes several lines spanning $3000-8000 \mathrm{~K}$ in upper level energy $E_{u}$, which are close to and overlap with the upper level energies of $\mathrm{H}_{2} \mathrm{O}$ $\left(E_{u}=8000-10,000 \mathrm{~K}\right)$ and $\mathrm{OH}\left(E_{u} \sim 5400-5700 \mathrm{~K}\right)$ at $2.9 \mu \mathrm{m}$ (Table 3 ). These lines therefore may share similar excitation conditions and emit from a similar disk region, as supported by previous observations of similar line profiles for $4.7 \mu \mathrm{m} \mathrm{CO}$ and $2.9 \mu \mathrm{m} \mathrm{H}_{2} \mathrm{O}$ and $\mathrm{OH}$ emission in a handful of disks (Salyk et al. 2008; Pontoppidan et al. 2010b; Banzatti et al. 2015; Brittain et al. 2016).
Table 3

Molecular Lines Properties

\begin{tabular}{|c|c|c|c|}
\hline $\begin{array}{l}\text { Wavelength } \\
(\mu \mathrm{m})\end{array}$ & Transition & $\begin{array}{c}A_{u l} \\
\left(\mathrm{~s}^{-1}\right)\end{array}$ & $\begin{array}{c}E_{u} \\
(\mathrm{~K})\end{array}$ \\
\hline \multicolumn{4}{|c|}{$\mathrm{H}_{2} \mathrm{O}$ Lines } \\
\hline 2.9273 & $15_{115} \rightarrow 16_{116}$ & 49 & 8744 \\
\hline 2.9278 & $12_{39} \rightarrow 13_{310}$ & 52 & 8388 \\
\hline 2.9291 & $13_{311} \rightarrow 14_{312}$ & 48 & 8583 \\
\hline 2.9292 & $14_{113} \rightarrow 15_{114}$ & 49 & 8698 \\
\hline 12.396 & $17_{413} \rightarrow 16_{314}$ & 7.7 & 5781 \\
\hline 12.407 & $16_{313} \rightarrow 15_{214}$ & 4.2 & 4945 \\
\hline 12.454 & $13_{76} \rightarrow 12_{49}$ & 1.2 & 4213 \\
\hline 12.519 & $15_{1213} \rightarrow 14_{114}$ & 1.5 & 4133 \\
\hline 12.596 & $13_{67} \rightarrow 12_{310}$ & 0.8 & 3966 \\
\hline 17.103 & $12_{58} \rightarrow 11_{29}$ & 3.7 & 3274 \\
\hline 17.225 & $11_{39} \rightarrow 10_{010}$ & 1.0 & 2439 \\
\hline 17.358 & $11_{29} \rightarrow 10_{110}$ & 0.9 & 2432 \\
\hline 30.525 & $7_{61} \rightarrow 6_{52}$ & 14 & 1750 \\
\hline 30.871 & $8_{54} \rightarrow 7_{43}$ & 8.7 & 1806 \\
\hline 32.991 & $7_{52} \rightarrow 6_{43}$ & 8.3 & 1525 \\
\hline 33.005 & $6_{60} \rightarrow 5_{51}$ & 13 & 1504 \\
\hline 63.323 & $8_{18} \rightarrow 7_{07}$ & 1.7 & 1070 \\
\hline \multicolumn{4}{|c|}{ OH Lines } \\
\hline 2.9343 & $3 / 2 \mathrm{P} 4.5 \mathrm{e}$ & 11.7 & 5400 \\
\hline 2.9346 & $3 / 2 \mathrm{P} 4.5 \mathrm{f}$ & 11.7 & 5400 \\
\hline 12.647 & $1 / 2 \mathrm{R} 22.5 \mathrm{ef}$ & 213 & 15100 \\
\hline 12.657 & $3 / 2$ R23.5ef & 213 & 15100 \\
\hline 30.277 & 3/2 R8.5f & 14.3 & 2380 \\
\hline 30.346 & $3 / 2$ R8.5e & 14.3 & 2380 \\
\hline \multicolumn{4}{|c|}{ CO Lines } \\
\hline 4.7545 & $v=1-0 \mathrm{P} 10$ & 17.3 & 3300 \\
\hline 4.7589 & $v=2-1 \mathrm{P} 4$ & 37.4 & 6160 \\
\hline
\end{tabular}

Note. Line properties are taken from the HITRAN 2012 database (Rothman et al. 2013).

\subsection{Photospheric Correction of $2.9 \mu \mathrm{m}$ Spectra}

We take each $2.9 \mu \mathrm{m}$ spectrum that has substantial stellar photospheric absorption and correct it by subtraction of the photospheric template that best matches its absorption spectrum. Given the limited grid of spectral-type templates obtained in this survey (Figure 2), the best matching template is usually easily identified for each science spectrum. We apply the following procedure to the spectral range between 2.925 and $2.935 \mu \mathrm{m}$, where the signal-to-noise ratio $(\mathrm{S} / \mathrm{N})$ is higher, the spectrum is less affected by detector or telluric gaps, and the $\mathrm{H}_{2} \mathrm{O}$ and $\mathrm{OH}$ lines are more isolated and stronger (Figure 2). The template spectrum is first shifted to compensate for velocity differences with the science target by cross-correlating the $\mathrm{H}_{2} \mathrm{O}$ and $\mathrm{OH}$ photospheric features in the science and template spectra. To match the science spectrum, the template is then artificially veiled to account for circumstellar disk emission and its absorption lines broadened. This is achieved by adding an excess flat continuum and by convolving the template spectrum with a Gaussian of variable width (for this technique, see also Hartigan et al. 1989, 1991; Basri \& Batalha 1990). The best fit is found by minimizing the residuals after subtraction of the veiled and broadened template from the science spectrum, over a grid of veiling and broadening values. Residuals are measured as the standard 


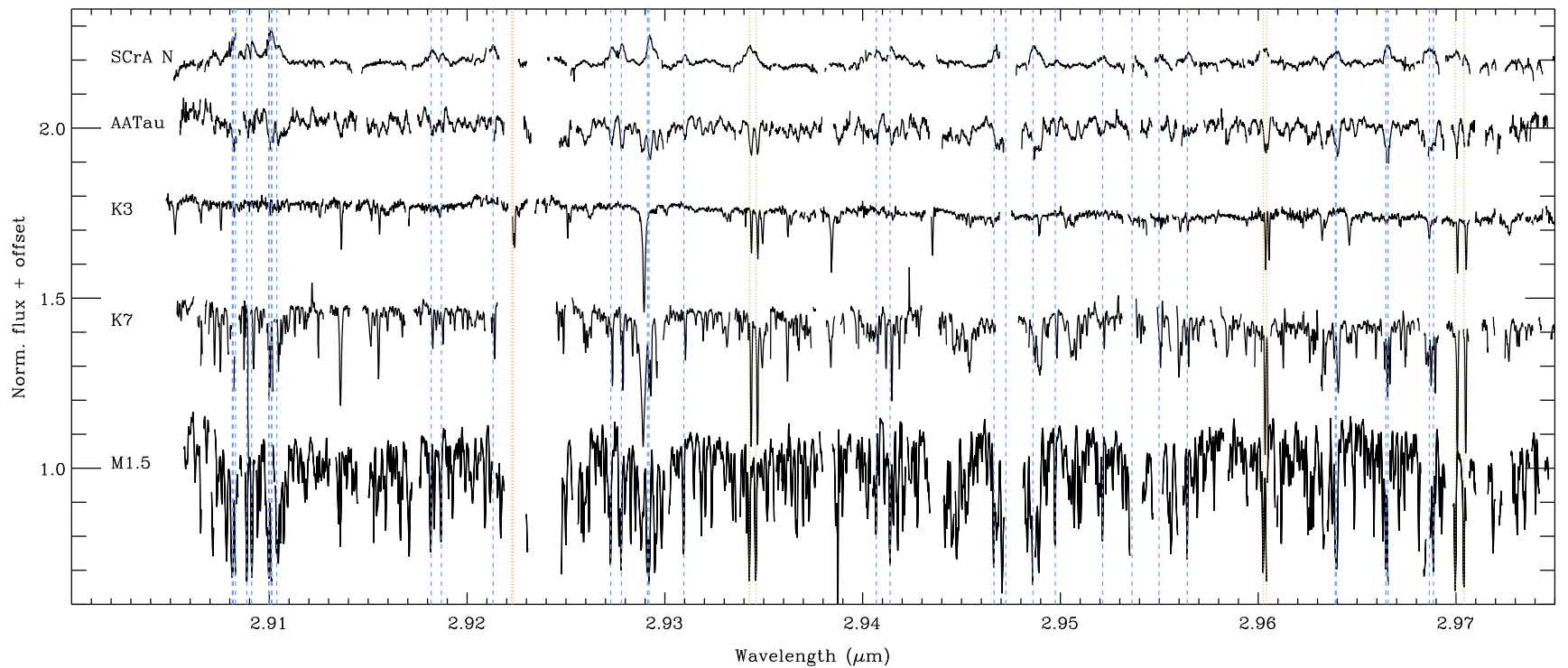

Figure 2. Examples of VLT-CRIRES spectra at $2.9 \mu \mathrm{m}$. From top to bottom: a disk spectrum in emission (SCrA N, a K3 star), a spectrum showing moderately veiled photospheric absorption (AA Tau, a K7 star), and the three photospheric templates used for correction of the CRIRES spectra (K3 type: NLTT 29176, K7 type: HD 111631, and M1.5 type: HIP 49986). Individual water transitions are marked with blue dashed lines, while OH transitions are marked with orange dotted lines.

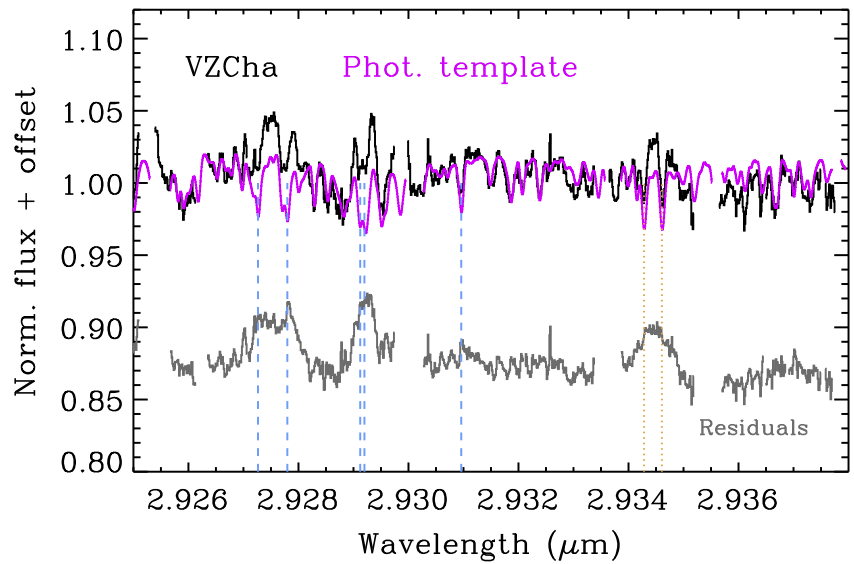

Figure 3. Example of the photospheric correction of protoplanetary disk spectra observed with CRIRES at $2.9 \mu \mathrm{m}$ (the spectra from the entire survey are shown in the Appendix). The spectrum is shown at the top in black, with its photospheric template in magenta (veiled and broadened). The molecular emission spectrum after correction is shown at the bottom in gray. Water and $\mathrm{OH}$ transitions are marked in blue and orange as in Figure 2.

deviation of pixel values from the median-smoothed de-veiled spectrum, in a spectral range free of disk molecular emission $(2.930-2.934 \mu \mathrm{m})$. Figure 3 shows an example of the photospheric correction procedure as applied to the spectrum of VZCha; plots for all the other targets are reported in the Appendix. Table 4 lists, for each target, the best photospheric standard match and the estimated veiling at $2.9 \mu \mathrm{m}$. Veiling measurements may be refined in future work by using a denser sampling of template spectral types.

\subsection{Characterization of $\mathrm{CO}$ Line Profiles}

The analysis of $\mathrm{CO}$ lines has been presented in Banzatti \& Pontoppidan (2015), and here we provide a short summary. We built high $\mathrm{S} / \mathrm{N}$ CO line profiles for each disk by stacking rovibrational transitions observed in the $4.7 \mu \mathrm{m}$ spectra. The stacked line profiles preserve the line flux as well as the line
Table 4

Photospheric Correction and Veiling of $2.9 \mu \mathrm{m}$ Disk Spectra

\begin{tabular}{lllcr}
\hline \hline Name & SpT & Phot. Templ. & $2.9 \mu \mathrm{m}$ Veil. & Note \\
\hline AATau & K7 & M1.5 & 2.2 & a \\
CWTau & K3 & (M1.5) & $(14.0)$ & a \\
DFTau & M1 & M1.5 & 2.0 & $\ldots$ \\
DOTau & M0 & M1.5 & 7.0 & $\ldots$ \\
EXLup14 & M0 & M1.5 & 2.7 & $\ldots$ \\
FNTau & M5 & M1.5 & 0.24 & b \\
GQLup & K7 & K7 & 4.8 & $\ldots$ \\
HTLup & K2 & (K7) & $(13.4)$ & a \\
IMLup & M0 & (M1.5) & $(1.7)$ & \\
RULup & K7 & M1.5 & 13.0 & $\ldots$ \\
SCrA S & M0 & M1.5 & 13.3 & $\ldots$ \\
TWCha & K7 & M1.5 & 4.4 & $\ldots$ \\
TWHya & K7 & M1.5 & 0.8 & b \\
VWCha & K5 & K7 & 5.4 & $\ldots$ \\
VZCha & K6 & M1.5 & 6.8 & $\ldots$ \\
WaOph6 & K6 & K7 & 2.2 & $\ldots$ \\
WXCha & M0 & M1.5 & 11.4 & $\ldots$ \\
\hline
\end{tabular}

Notes.

${ }^{\text {a }}$ Residual photospheric absorption.

${ }^{b}$ Possible emission as narrow as photospheric lines. See the Appendix for details.

shape, through a homogeneous analysis that we adopted for the entire disk sample. We stacked those lines that are most commonly available across the sample (due to the observing strategies and the detector characteristics), that have similar flux, and that are not blended with higher-level transitions. These requirements led us to typically use the $v=1-0$ lines between $\mathrm{P} 7$ and $\mathrm{P} 12$, and the $v=2-1$ lines between $\mathrm{P} 3$ and P6. Each line was normalized to its local continuum, resampled on a common velocity grid, and stacked by weighted average.

The $v=1-0$ and $v=2-1$ stacked line profiles were then used to identify and separate multiple emission components, where present. The $v=2-1$ lines, with higher $E_{u}$ (Table 3), 

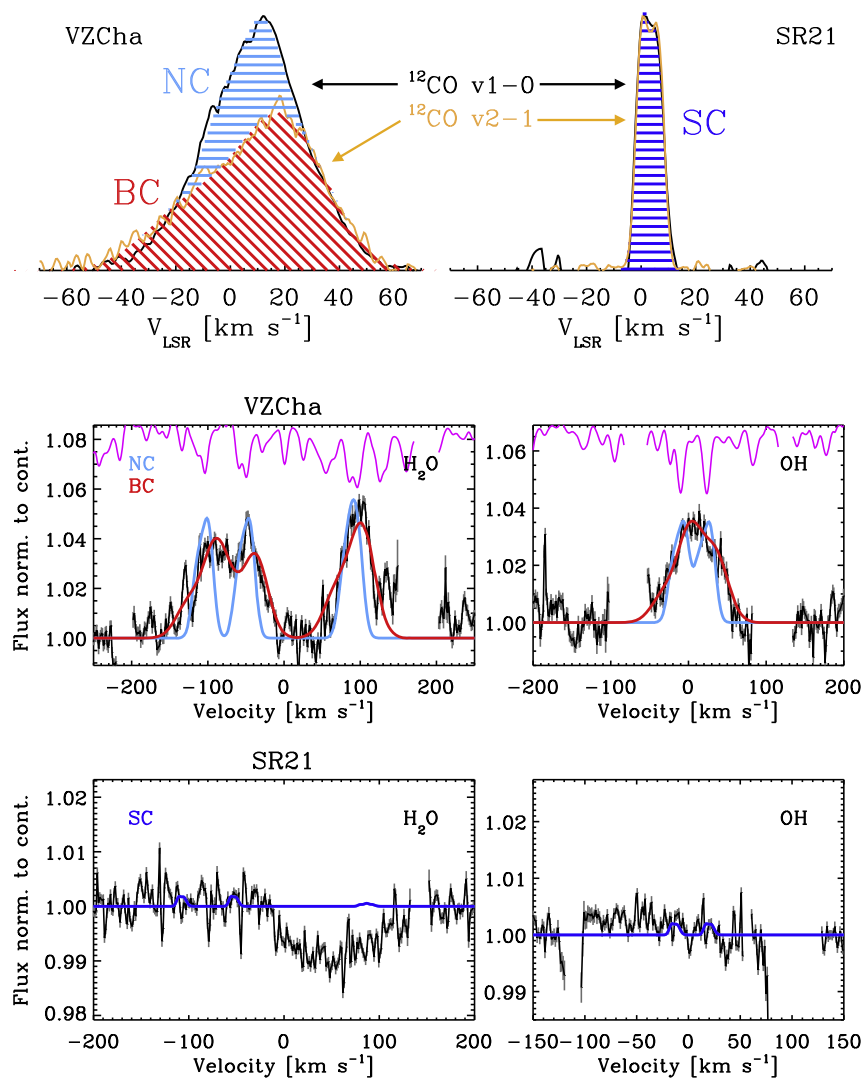

Figure 4. Top: Spectral decomposition of $\mathrm{CO}$ lines profiles, as presented in Banzatti \& Pontoppidan (2015). The spectrum of VZCha is an example of a "double-component disk," where CO rovibrational emission at $4.7 \mu \mathrm{m}$ has two velocity components (the broad component $\mathrm{BC}$ marked in red; the narrow component NC marked in cyan). The spectrum of SR21 shows an example of a "single-component disk" disk (SC), where only a narrow CO component is found. The gallery of $\mathrm{CO}$ line profiles is shown in Banzatti \& Pontoppidan (2015). Center and bottom: examples of fits to $\mathrm{H}_{2} \mathrm{O}$ and $\mathrm{OH}$ line profiles at $2.93 \mu \mathrm{m}$ by using the $\mathrm{CO}$ emission components (fits to other disks are shown in the Appendix). The spectrum is shown in black with gray error bars, centered in velocity on (for $\mathrm{OH}$ ) or in between (for $\mathrm{H}_{2} \mathrm{O}$ ) the lines for visualization. The best-fit line profiles obtained by combining $\mathrm{CO}$ lines are shown in cyan (using the $\mathrm{NC}$ ), red (using the $\mathrm{BC}$ ), and blue for the single-component disk. The photospheric template, where used to remove the stellar photosphere, is shown at the top in magenta for reference.

are mostly excited in the hotter innermost disk region, while the $v=1-0$ lines, with lower $E_{u}$, often show additional flux contributions from colder gas at larger disk radii. This has been empirically demonstrated through the systematic decomposition of CO lines, where a broad (inner) component "BC" is identified by the $v=2-1$ lines that match the wings of $v=1-0$ lines, and a narrow (outer) component "NC" is obtained from the residuals by subtraction of the $v=2-1$ profile (which is dominated in flux by BC) from the $v=1-0$ profile (which includes both BC and NC). An example of this procedure is included in Figure 4. In several disks, the two velocity components are clearly seen by visual inspection of the $v=1-0$ lines, which show a narrower peak on top of broader wings (Bast et al. 2011; Herczeg et al. 2011; Banzatti \& Pontoppidan 2015). Those disks that, following this analysis, have been found to have only one $\mathrm{CO}$ component (i.e., those where the $v=1-0$ and $v=2-1$ stacked line profiles match) have been identified as "single-component" disks ("SC"; e.g., SR21 in Figure 4).
Table 5

Summary of 2.9-4.7 $\mu \mathrm{m}$ Emission Detections and Properties

\begin{tabular}{lclccc}
\hline \hline Component & $\begin{array}{c}\text { FWHM } \\
\left(\mathrm{km} \mathrm{s}^{-1}\right)\end{array}$ & $\begin{array}{c}R_{\mathrm{co}} \\
(\mathrm{au})\end{array}$ & \multicolumn{2}{c}{$\begin{array}{c}\mathrm{CO} \\
\text { (detection fractions) }\end{array}$} \\
\hline Broad (BC) & $50-200$ & $0.04-0.3$ & $25 / 25$ & $15 / 20$ & $17 / 20$ \\
Narrow (NC) & $10-70$ & $0.2-3$ & $23 / 25$ & $3 / 20$ & $3 / 20$ \\
Single (SC) & $6-70$ & $0.3-20$ & $28 / 28$ & $1 / 10$ & $4 / 10$ \\
\hline
\end{tabular}

Note. The definition of components in the first column is based on the analysis of $4.7 \mu \mathrm{m} \mathrm{CO}$ emission in Banzatti \& Pontoppidan (2015), as summarized in Section 3.2. Details on individual sources are given in the Appendix.

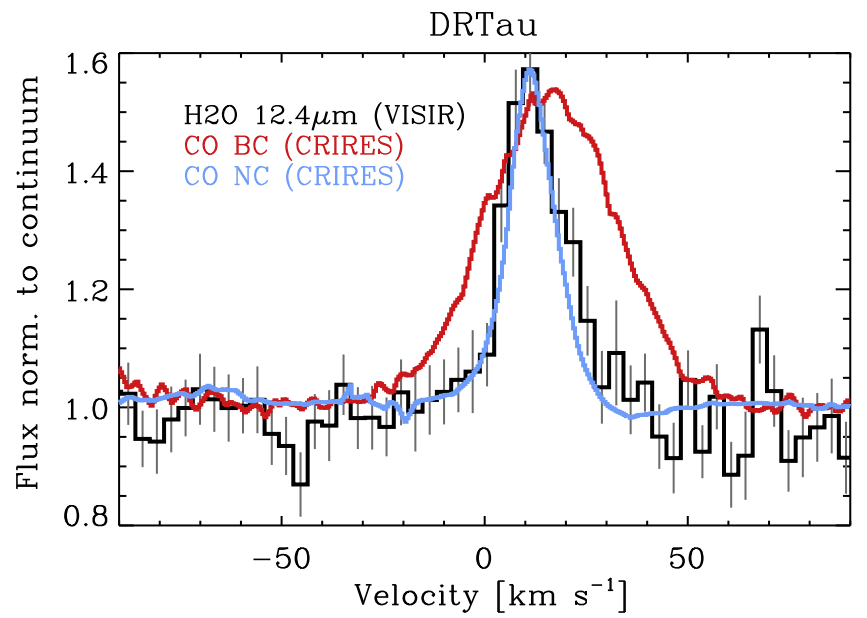

Figure 5. $\mathrm{H}_{2} \mathrm{O}$ line at $12.396 \mu \mathrm{m}$ as observed in DRTau with VLT-VISIR at a resolution of $\sim 15 \mathrm{~km} \mathrm{~s}^{-1}$ (Banzatti et al. 2014). The rovibrational CO line profiles of the broad and narrow components are shown in red and cyan, scaled to the VISIR line peak for comparison.

\subsection{Characterization of $\mathrm{H}_{2} \mathrm{O}$ and $\mathrm{OH}$ Line Profiles}

We use the stacked $\mathrm{CO}$ line profiles to reproduce the observed $\mathrm{H}_{2} \mathrm{O}$ and $\mathrm{OH}$ emission features, to test if their shapes and width are equal to those of $\mathrm{CO}$, implying that they emit from a similar disk region. We adopt the $\mathrm{CO}$ line profile at each rest wavelength of $\mathrm{H}_{2} \mathrm{O}$ and $\mathrm{OH}$ transitions, combine them into a single feature if the lines are broad and overlap (which typically happens for the broad component), and then vary the peak flux of the combined line profile to fit the $2.9 \mu \mathrm{m}$ spectrum (see Figure 4 and the Appendix).

$\mathrm{H}_{2} \mathrm{O}$ and $\mathrm{OH}$ lines at $2.9 \mu \mathrm{m}$ are typically detected in doublecomponent disks only (i.e., when the $\mathrm{BC}$ is not detected in $\mathrm{CO}$, $\mathrm{H}_{2} \mathrm{O}$ and $\mathrm{OH}$ at $2.9 \mu \mathrm{m}$ are typically not detected either). Where detected, $\mathrm{H}_{2} \mathrm{O}$ and $\mathrm{OH}$ lines at $2.9 \mu \mathrm{m}$ are dominated by a broad component that matches the $\mathrm{CO}$ BC profile (Figure 4 and Table 5); we therefore conclude that these lines are emitted at similar disk radii. This was first recognized in the 3-5 $\mu \mathrm{m}$ spectrum of EX Lupi by Banzatti et al. (2015), and applies to the majority of disks in the sample studied here (see Table 5). Disks that lack the $\mathrm{CO}$ broad component (the "singlecomponent" disks) do not show $\mathrm{H}_{2} \mathrm{O}$ emission at $2.9 \mu \mathrm{m}$ either. This is the first hint that disks with evidence for an inner $\mathrm{CO}$-depleted region are also depleted in water, which will be further detailed and discussed in the next sections. In these disks, $\mathrm{OH}$ is detected in a few cases. This finding agrees with previous work that detected $\mathrm{OH}$ but not $\mathrm{H}_{2} \mathrm{O}$ in some disks 


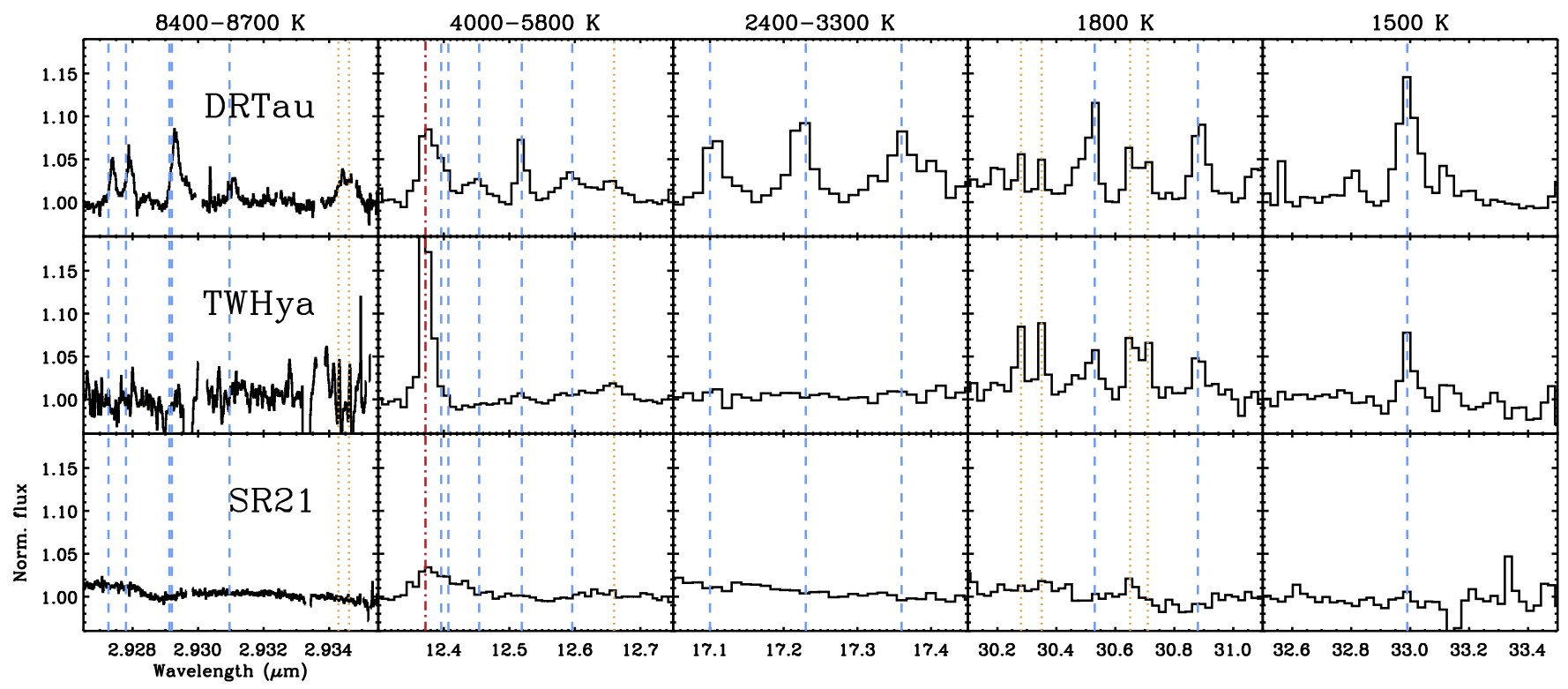

Figure 6. Representative samples of water vapor emission from 2.9 to $35 \mu \mathrm{m}$, combining observations from VLT-CRIRES and Spitzer-IRS. Individual water transitions are marked with blue dashed lines, while $\mathrm{OH}$ transitions are marked with orange dotted lines. The HI 7-6 line is marked in a red dashed-dotted line to avoid confusion with water emission. Increasing wavelengths probe colder water transitions (the range in upper level energy in each spectral sample is displayed at the top of the figure; see also Table 3).

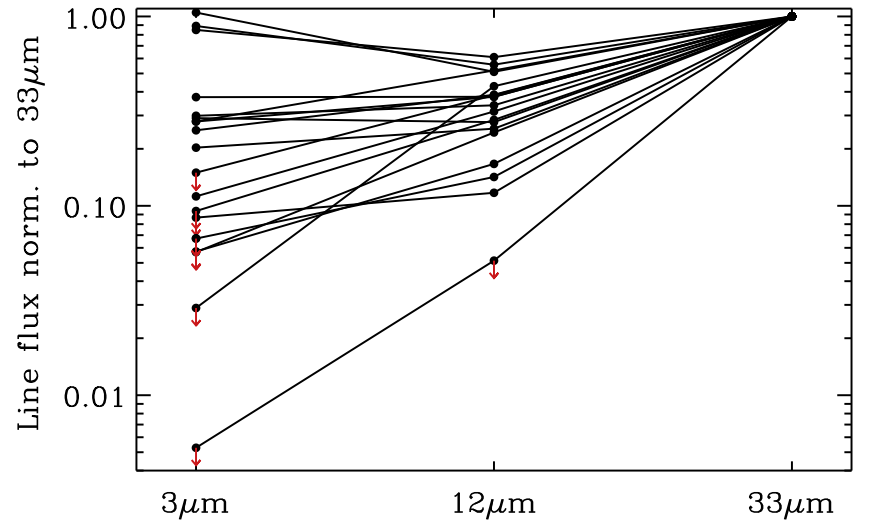

Figure 7. Measured $\mathrm{H}_{2} \mathrm{O}$ line fluxes in disks that have both CRIRES and Spitzer spectra available. Line fluxes from each disk are linked with a line and are normalized to their $33 \mu \mathrm{m}$ line flux (the lowest-energy line that we include in the analysis; see Table 3). Upper limits are shown with red arrows. The wavelength dependence of the decrease in the line fluxes is found both in the line-to-continuum strength, illustrated for three disks in Figure 6, and in the integrated line fluxes, as shown here.

around intermediate-mass stars (Mandell et al. 2008; Fedele et al. 2011); disks in this stellar mass bin are in fact found to typically have only a single, narrow CO component (Banzatti \& Pontoppidan 2015).

While $\mathrm{H}_{2} \mathrm{O}$ and $\mathrm{OH}$ emission at $2.9 \mu \mathrm{m}$ comes from similar disk radii as the $\mathrm{CO}$ broad component $(\sim 0.04-0.3 \mathrm{au})$, a survey of velocity-resolved $\mathrm{H}_{2} \mathrm{O}$ and $\mathrm{OH}$ emission at $>2.9 \mu \mathrm{m}$ is still missing, and is essential to determine which disk radii are probed at longer wavelengths. $\mathrm{H}_{2} \mathrm{O}$ emission at $12.4 \mu \mathrm{m}$ may come from a region at larger disk radii that matches the emitting region of the narrow $\mathrm{CO}$ component, as shown in Figure 5 for one disk. In fact, the $12.4 \mu \mathrm{m} \mathrm{H}_{2} \mathrm{O}$ lines have lower energy than the $2.93 \mu \mathrm{m}$ lines (Table 3), and may therefore match the colder/outer component of $\mathrm{CO}$ emission rather than the hotter/inner one.

\section{LINE FLUXES ACROSS DISK RADII}

By combining $\mathrm{H}_{2} \mathrm{O}$ and $\mathrm{OH}$ spectra between 2.9 and $35 \mu \mathrm{m}$, we find a wavelength dependence in the decrease of line fluxes. When the emission is detected at $2.9 \mu \mathrm{m}$, it is always detected at longer wavelengths, up to $33 \mu \mathrm{m}$. On the contrary, when lines at $30-33 \mu \mathrm{m}$ are detected, those at 12 or $2.9 \mu \mathrm{m}$ may not necessarily be (see Figures 6 and 7, and Table 6). The decrease of water emission is found as a sequential intrinsic decrease of the observed line fluxes and of the line-to-continuum strength, where water line fluxes at short wavelengths decrease when water line fluxes at longer wavelengths are still strong. Since the emission probes hotter to colder gas from short to long wavelengths (following a global trend in $E_{u}$ energies that decrease with increasing wavelength; see Table 3, Figure 6, and Blevins et al. 2016), this wavelength dependence of the line flux decrease may reflect the physical removal of molecular gas in the disk from the inside out (as found in the disk of TW Hya; Zhang et al. 2013).

In Section 3, we reported that $\mathrm{H}_{2} \mathrm{O}$ and $\mathrm{OH}$ emission at $2.9 \mu \mathrm{m}$ comes from a disk region that is consistent with that of the broad CO emission at $4.7 \mu \mathrm{m}$, so that we can adopt $R_{\mathrm{co}}$ as descriptive of $\mathrm{H}_{2} \mathrm{O}$ and $\mathrm{OH}$ emission, too. The fact that the high-energy $\mathrm{H}_{2} \mathrm{O}$ lines at $2.9 \mu \mathrm{m}$ disappear when the broad component of $\mathrm{CO}$ emission disappears from the observed spectra suggests that the depletion of the hotter/inner $\mathrm{CO}$ gas and the depletion of the hotter/inner $\mathrm{H}_{2} \mathrm{O}$ gas proceed together. We now combine measurements from Banzatti \& Pontoppidan (2015) and this work to test the dependence of $\mathrm{H}_{2} \mathrm{O}$ and $\mathrm{OH}$ line fluxes on $R_{\mathrm{co}}$ as a tracer of molecular gaps in inner disks.

\subsection{Relations Between Line Fluxes and Gap Size}

$R_{\text {co }}$ measures the location of $\mathrm{CO}$ emission in the disk by taking into account the stellar mass and disk inclination, which are both linked to the measured line FWHM through Kepler's law as

$$
R_{\mathrm{co}}=(2 \sin i / \mathrm{FWHM})^{2} G M_{\star}
$$



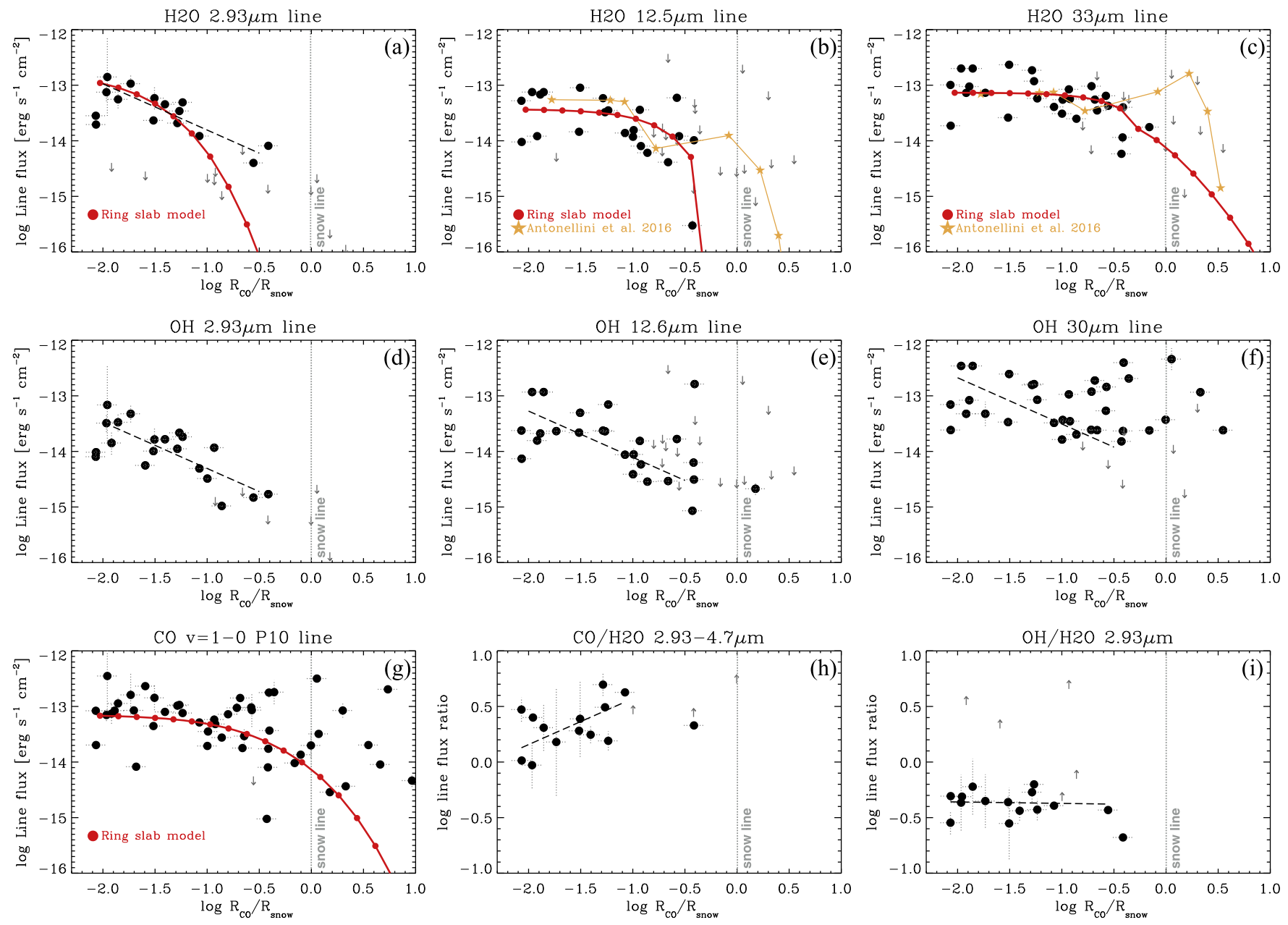

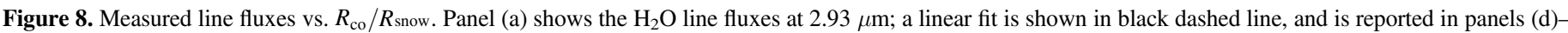

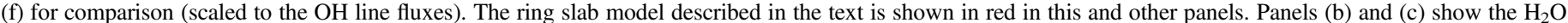

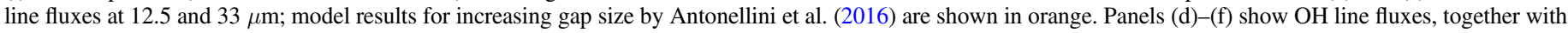

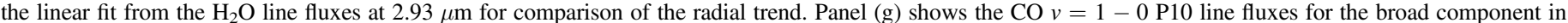

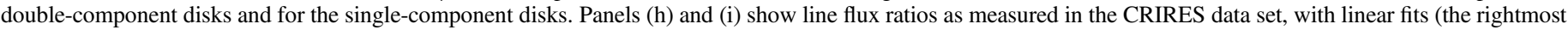

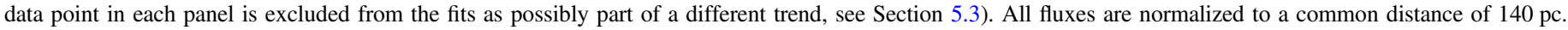

where $M_{\star}$ is the stellar mass, $i$ is the the disk inclination, and $G$ is the gravitational constant, and we take the line velocity at the half width at half maximum (FWHM/2), as in Banzatti \& Pontoppidan (2015). As a reference comparison for $R_{\text {co }}$, we take the midplane water snow line radius $R_{\text {snow }}$, defined as

$$
\begin{aligned}
R_{\text {snow }}= & 2.1 \text { au }\left(M_{\star} / M_{\odot}\right)^{1 / 3}\left(M_{\text {acc }} / 10^{-8} M_{\odot} \mathrm{yr}^{-1}\right)^{4 / 9} \\
& \times\left(T_{\text {snow }} / 160 \mathrm{~K}\right)^{-10 / 9}
\end{aligned}
$$

where $M_{\text {acc }}$ is the accretion rate and $T_{\text {snow }}$ is the water freezeout temperature. Equation (2) is the midplane snow line radius for a viscously heated disk as derived by Mulders et al. $(2015)^{8}$, who used $T_{\text {snow }}=160 \mathrm{~K}$ as in Min et al. (2011). This formula assumes that the disk midplane heating by stellar irradiation is negligible, and this has been shown to be valid for disks around stars younger than $\sim 3 \mathrm{Myr}$ with mass up to $\sim 2 M_{\odot}$ and accretion rates between $10^{-9}$ and $10^{-6} M_{\odot} \mathrm{yr}^{-1}$ (Kennedy \& Kenyon 2008; Min et al. 2011; Mulders et al. 2015). Some

\footnotetext{
8 We assume a gas-to-dust ratio of 100 , an $\alpha$ viscosity parameter of 0.01 , and a Rosseland mean dust opacity of $770 \mathrm{~cm}^{2} \mathrm{~g}^{-1}$ that normalize the extra terms in Equation (2) as formulated in Mulders et al. (2015).
}

disks in our sample exceed these limits in stellar mass and age, while some have inner dust gaps that may change the disk temperature structure. For simplicity we adopt the same equation for the entire sample. By normalizing to $R_{\text {snow }}$, we put $R_{\text {co }}$ from individual disks in reference to a disk radius of equal temperature across the entire disk sample, as set by water ice evaporation in the disk midplane. $R_{\mathrm{co}} / R_{\mathrm{snow}}$ therefore enables a comparison of results from individual disks within a common reference framework.

Figure 8 shows that line fluxes of $\mathrm{H}_{2} \mathrm{O}, \mathrm{OH}$, and $\mathrm{CO}$ decrease with increasing $R_{\mathrm{co}} / R_{\text {snow }}$, i.e., as the inner gap radius increases and reaches the snow line radius. ${ }^{9} \mathrm{H}_{2} \mathrm{O}$ line fluxes at $2.9 \mu \mathrm{m}$ show a decreasing linear correlation at radii $-2 \lesssim \log R_{\text {co }} / R_{\text {snow }} \lesssim-0.5$, beyond which they fall under the detection limit $\left(\sim 2 \times 10^{-15} \mathrm{erg} \mathrm{s}^{-1} \mathrm{~cm}^{-2}\right.$ distance/ $140 \mathrm{pc}$ ). A linear fit to the detected lines provides (using the

\footnotetext{
Snow line radii following Mulders et al. (2015) are on average $\lesssim 1.5$ times larger than those formulated by Kennedy \& Kenyon (2008) at stellar masses between 1 and $3 M_{\odot}$; while the $R_{\text {co }} / R_{\text {snow values slightly shift depending on the }}$ formula used for the snow line radius, the global trends presented here are preserved.
} 

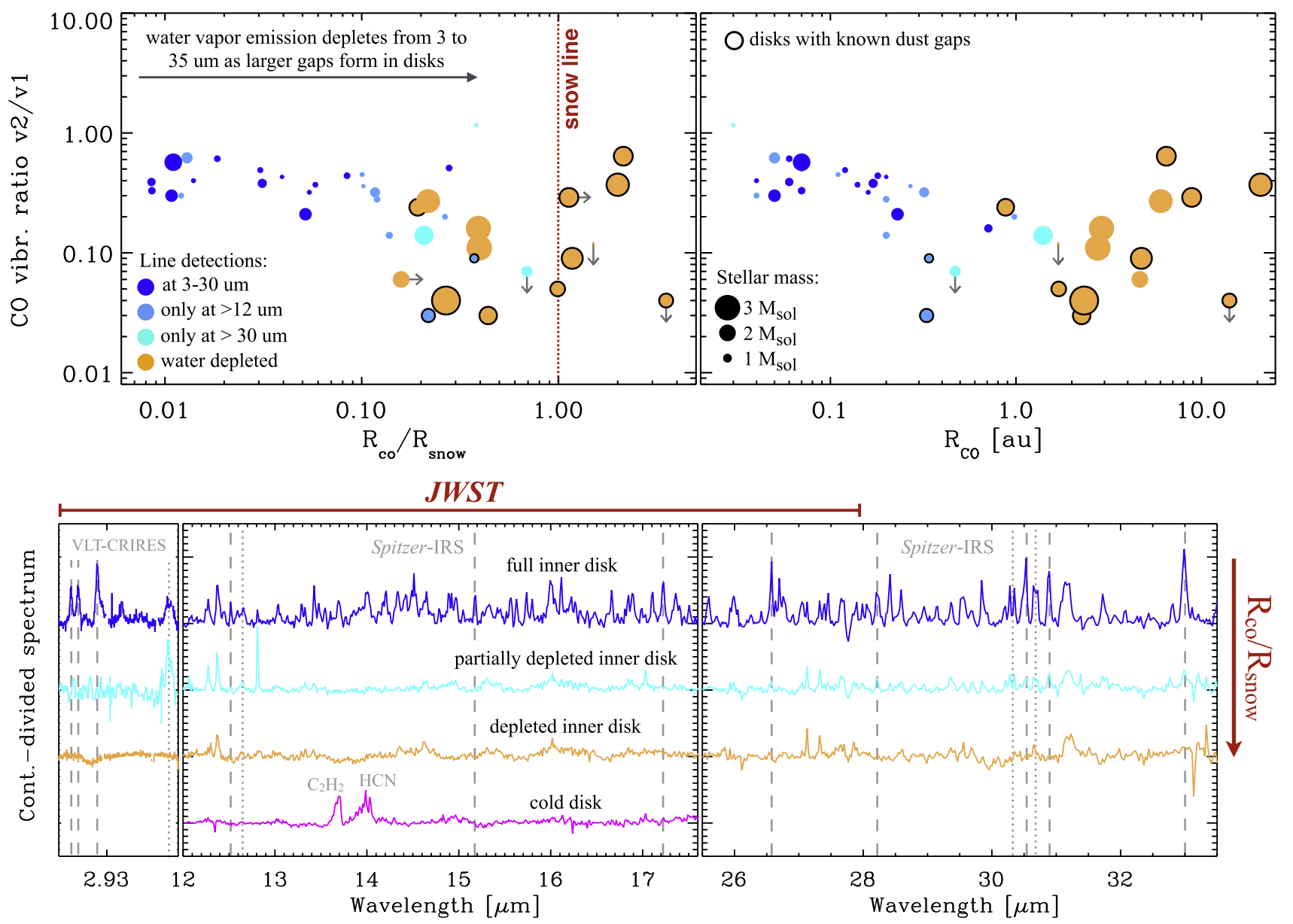

Figure 9. Inside-out water vapor depletion from 2.9-33 $\mu \mathrm{m}$ spectra of protoplanetary disks. Top: the data points show the CO sequence in the temperature-radius diagram of CO emission at $4.7 \mu \mathrm{m}$ from Banzatti \& Pontoppidan (2015), normalized to the midplane snow line radius given by Equation (2) in the left plot. The data are color-coded according to water detections (line fluxes are included in Table 6). The size of data points is proportional to the stellar mass as shown, and disks where dust gaps have been found in previous work are marked with a black circle. Bottom: three steps along the $\mathrm{CO}$ and $\mathrm{H}_{2} \mathrm{O}$ depletion sequence are visualized using averaged Spitzer spectra: (i) water emission is strong at 2.9-33 $\mu \mathrm{m}$ in disks when $R_{\mathrm{co}} / R_{\text {snow }} \lesssim 0.1$, (ii) water emission is weaker but still detected at 25-33 $\mu \mathrm{m}$ in disks when $0.1 \lesssim R_{\text {co }} / R_{\text {snow }} \lesssim 1$, and (iii) water emission is undetected at all infrared wavelengths $2.9-33 \mu \mathrm{m}$ when $R_{\mathrm{co}} / R_{\text {snow }} \gtrsim 1$. The averaged Spitzer spectrum of disks around low-mass stars $\left(M_{\star}<0.2 M_{\odot}\right)$ is shown at the bottom for comparison. Some prominent water and $\mathrm{OH}$ emission lines are marked respectively with dashed and dotted lines for reference.

Bayesian method by Kelly 2007):

$$
\operatorname{Flux}_{140}\left(\mathrm{H}_{2} \mathrm{O}\right)=-14.6 \pm 0.2+\left(\mathrm{R}_{\text {co }} / \mathrm{R}_{\text {snow }}\right)^{-0.8 \pm 0.2}
$$

where Flux $_{140}$ is the measured line flux normalized to a distance of 140 pc. At 12.5 and $33 \mu \mathrm{m}, \mathrm{H}_{2} \mathrm{O}$ lines are detected out to $\log R_{\mathrm{co}} / R_{\text {snow }} \sim-0.2$, and decrease only at $\log R_{\mathrm{co}} /$ $R_{\text {snow }} \gtrsim-1$. OH emission follows similar trends, but $\mathrm{OH}$ lines are tentatively detected up to larger disk radii (especially the cold lines at $30 \mu \mathrm{m}$ ). Variability in accretion luminosity may contribute to the order-of-magnitude scatter in line fluxes along these trends; however, a factor up to $\approx 10$ change in line fluxes has been observed so far only in the extreme accretion outburst of EX Lupi, and not during the typically lower variability of other young stars included in this sample (Banzatti et al. 2012, 2014, 2015). Similarly to $\mathrm{H}_{2} \mathrm{O}$ and $\mathrm{OH}$, the $\mathrm{CO}$ $v=1-0$ line flux decreases with $R_{\mathrm{co}} / R_{\text {snow }}$, but it is detected in disks well beyond the snow line radius. We interpret this as evidence that what regulates the observed $\mathrm{H}_{2} \mathrm{O}$ and $\mathrm{OH}$ emission is the molecular gas left within the snow line radius, or in other words that $\mathrm{H}_{2} \mathrm{O}$ and $\mathrm{OH}$ emission disappears as inner disks are depleted from the inside out to the snow line (i.e., as $R_{\text {co }}$ grows larger and approaches $R_{\text {snow }}$ ), beyond which the reservoir of water vapor is strongly limited due to freezeout at low temperatures. $\mathrm{CO}$ gas is instead still detected beyond the snow line because it freezes out at lower temperatures $(\sim 20 \mathrm{~K})$, found at larger disk radii.

As a basic test of this inside-out depletion scenario, we use a simple disk model composed of concentric rings of increasing radius, where each ring is a slab of gas described by one temperature and one column density as in Banzatti et al. (2012). We adopt power-law profiles to set how the temperature and column density of each ring decrease with increasing disk radius, as $T(\mathrm{R})=T_{0}\left(R / R_{0}\right)^{-q}$ and $N(\mathrm{R})=N_{0}\left(R / R_{0}\right)^{-p}$. We calibrate the model using the observed CO line fluxes, by adopting model parameter values from the radial temperature profile measured by Banzatti \& Pontoppidan (2015) in rovibrational $\mathrm{CO}$ emission, as $T_{0}=1300 \mathrm{~K}, q=0.3$, $N_{0}=10^{20} \mathrm{~cm}^{-2}$, and $p=1$. In Figure 8 (panel $(\mathrm{g})$ ) we show how the integrated $\mathrm{CO}$ line flux from the model decreases with increasing hole size, by removing one ring at a time from $R_{0}=0.03$ au to $R_{\text {out }}=100$ au to mimic an inside-out removal of hotter to colder gas (the radial grid of disk rings is shown by the dots). A radially decreasing power-law temperature profile 


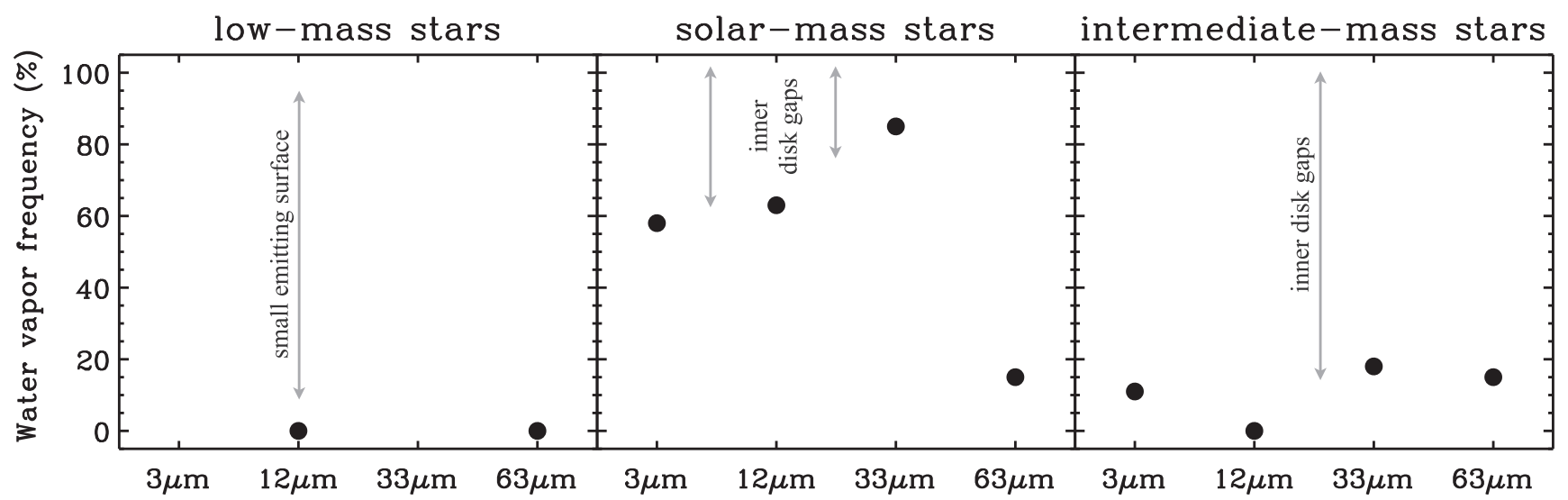

Figure 10. Detection rates of infrared water vapor emission for three stellar mass bins: low-mass stars and Brown Dwarfs $\left(<0.2 M_{\odot}\right)$, solar-mass stars $\left(0.2-1.5 M_{\odot}\right)$, and intermediate-mass stars $\left(1.5-4 M_{\odot}\right)$. Results from VLT-CRIRES, Spitzer-IRS, and Herschel-PACS surveys as reported in Table 1 are shown as black dots.
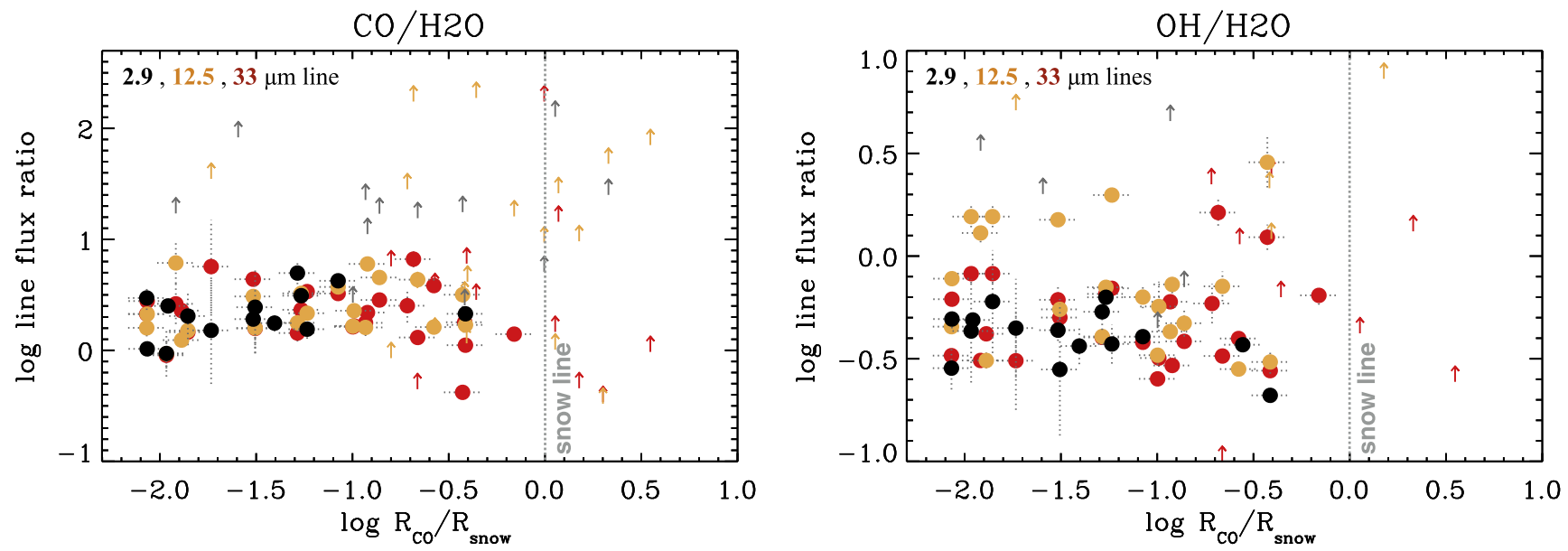

Figure 11. Left: $\mathrm{CO} / \mathrm{H}_{2} \mathrm{O}$ line flux ratio as measured using the $\mathrm{P} 10$ line for $\mathrm{CO}$ and the $2.93 \mu \mathrm{m}$ (black), $12.5 \mu \mathrm{m}$ (orange), and $33 \mu \mathrm{m}$ (red) lines for $\mathrm{H}_{2} \mathrm{O}$. Right: $\mathrm{OH} / \mathrm{H}_{2} \mathrm{O}$ ratio as measured at $2.9-33 \mu \mathrm{m}$.

reproduces the decrease in the $\mathrm{CO}$ line fluxes as a curve, where as the hole radius increases, colder gas emission beyond the hole produces weaker $\mathrm{CO}$ lines at $4.7 \mu \mathrm{m}$. CO line fluxes that exceed the model at $\log R_{\mathrm{co}} / R_{\text {snow }} \gtrsim-0.5$ come from disks where the emission is pumped by UV radiation (Brittain et al. 2003; Banzatti \& Pontoppidan 2015).

The same ring slab model is then used for $\mathrm{H}_{2} \mathrm{O}$ emission. Such a model produces line fluxes stronger than observed at 12.5 and $33 \mu \mathrm{m}$. We therefore include a step function for the column density, to simulate the presence of a radius in the surface of the disk beyond which water vapor is reduced by freeze-out or by chemistry in cold regions (e.g., van Dishoeck et al. 2013). We keep $p=1$ as used for $\mathrm{CO}$ at disk radii smaller than $\approx 2.5$ au, and adopt $p=2.5$ to produce a steeper decrease in water column density at larger disk radii (where the gas temperature decreases to $\lesssim 300 \mathrm{~K}$ ). This model, shown in panels (a), (b), and (c) of Figure 8, approximately reproduces both the absolute fluxes and the wavelength-dependent radial decrease in water emission at 2.93-33 $\mu \mathrm{m}$. A step function in the column density produces a decrease/cutoff in the 12.5 and $33 \mu \mathrm{m}$ line fluxes at $\log R_{\mathrm{co}} / R_{\text {snow }} \sim-0.5$, which is consistent with the observed water line fluxes in the Spitzer spectra.

The purpose of the ring slab model is to test in the simplest terms if an inside-out removal of disk gas can explain the observed line flux decrease within the snow line. Clearly, this simple model ignores several important factors that should be considered, such as a realistic physical disk structure, disk chemistry, and their adaptation to the formation and development of disk gaps. However, the model seems to have the key ingredients needed to qualitatively reproduce the observed data, by including a radially decreasing temperature and the insideout removal of molecular gas. Detailed modeling of the water spectrum and of its dependence on disk regions of water vapor depletion at and beyond the snow line has been presented by Meijerink et al. (2009), Zhang et al. (2013), Blevins et al. (2016), Notsu et al. (2016). Recent explorations by Antonellini et al. (2016) for a disk around an intermediate-mass star found a wavelength-dependent decrease in the Spitzer water line fluxes for a grid of increasing inner disk hole sizes, in qualitative agreement with what we measure in the data out to the snow line radius (Figure 8, panels (b) and (c)). These models produce an excess flux when the gap reaches the snow line radius, contrarily to the observations (see Section 5.3).

The wavelength-dependent decrease in water vapor emission between 2.9 and $33 \mu \mathrm{m}$ is summarized in Figure 9, where we show the temperature-radius (T-R) diagram originally defined for $\mathrm{CO}$ rovibrational emission by Banzatti \& Pontoppidan (2015) and we color code the individual disks by their $\mathrm{H}_{2} \mathrm{O}$ detections. For $R_{\mathrm{co}} / R_{\text {snow }}$ between 0.1 and 1 , all disks have lost their detectable $\mathrm{H}_{2} \mathrm{O}$ emission at $2.9 \mu \mathrm{m}$ (their line fluxes are 
$\lesssim 10^{-15} \mathrm{erg} \mathrm{s}^{-1} \mathrm{~cm}^{-2}$ distance $/ 140 \mathrm{pc}$ ), while some still retain detectable emission at 12 or $33 \mu \mathrm{m}$. Beyond the snow line $\left(R_{\text {co }} / R_{\text {snow }}=1\right), \mathrm{H}_{2} \mathrm{O}$ emission between 2.9 and $35 \mu \mathrm{m}$ is not detected in any disk. As a diagnostic of $R_{\mathrm{co}} / R_{\mathrm{snow}}$, we illustrate in Figure 9 the wavelength-dependent $\mathrm{H}_{2} \mathrm{O}$ emission decrease shown earlier in Figure 6, here in reference to the increasing disk gap size.

\subsection{Molecular Line Flux Ratios in Inner Disks}

Figure 8 (panels (h) and (i)) shows molecular line flux ratios as measured in the CRIRES data set $(2.9-4.7 \mu \mathrm{m})$. We find a constant $\mathrm{OH} / \mathrm{H}_{2} \mathrm{O}$ ratio of $\sim 0.4$ in the range $-2 \lesssim$ $\log \mathrm{R}_{\mathrm{co}} / R_{\text {snow }} \lesssim-0.5$. As for the $\mathrm{CO} / \mathrm{H}_{2} \mathrm{O}$ ratio, we find an increasing trend with increasing radius in the range $-2 \lesssim \log \mathrm{R}_{\text {co }} / R_{\text {snow }} \lesssim-1$. This trend is due to a decreasing $\mathrm{H}_{2} \mathrm{O}$ line flux, while the $\mathrm{CO}$ line flux is less steeply decreasing over these disk radii (Figure 8, panels (a) and (g)). By fitting the measured line ratios, we find the following linear relation (using Kelly 2007):

$$
\operatorname{Flux}(\mathrm{CO}) / \operatorname{Flux}\left(\mathrm{H}_{2} \mathrm{O}\right)=1.0 \pm 0.4+\left(\mathrm{R}_{\mathrm{co}} / \mathrm{R}_{\text {snow }}\right)^{0.4 \pm 0.2} \text {. }
$$

These trends are further analyzed and discussed in Section 5.3.

\section{DISCUSSION}

\subsection{Water Vapor and the Depletion of Inner Disks}

Water emission from protoplanetary disks has been detected by the Spitzer-IRS in more than 60 disks (see Section 1.1). This sample revealed two major trends: (1) water line fluxes increase with increasing stellar luminosity (and mass; see Figures 1 and 20, and Salyk et al. 2011a), and in contrast with the first trend, (2) the water vapor frequency is much higher in disks around stars of $0.2<M_{\star}<1.5 M_{\odot}$ (with a frequency of $60 \%-80 \%$ ) than in disks around stars of $M_{\star}>1.5 M_{\odot}$ (with a frequency of $<20 \%$; see Table 1, Figure 10, and Pontoppidan et al. 2010a). Recent chemical modeling of the molecular content of inner disks by Walsh et al. (2015), while able to reproduce the luminosity trend, was still unable to reproduce the absence of water emission in disks around intermediate-mass stars. We have demonstrated in Sections 3 and 4 that the strength of the infrared water spectrum is linked to an inside-out depletion scenario in disks by finding (1) a similar emitting region and detection frequency for the high-energy $\mathrm{H}_{2} \mathrm{O}$ lines at $2.9 \mu \mathrm{m}$ and the innermost CO gas (the broad component), (2) a wavelength-dependent decrease of water line fluxes, where hotter emission (as probed at $2.9 \mu \mathrm{m}$ ) decreases before colder emission (as probed at $33 \mu \mathrm{m}$ ), and (3) a correlation between the decrease of water line fluxes at $2.9-33 \mu \mathrm{m}$ and the increase in size of an inner molecular hole measured from $\mathrm{CO}$ emission.

One major implication of this analysis is that the low frequency of water vapor detections in disks around $M_{\star}>1.5 M_{\odot}$ is found to be linked to inner disk regions depleted in molecular gas out to or even beyond the water snow line, differently from most disks around solar-mass stars. Other independent techniques find signatures of inner holes in dust emission in a growing number of disks around intermediate-mass stars (e.g., Maaskant et al. 2013; Menu et al. 2015). With the analysis of disks that have CO emission observed at $4.7 \mu \mathrm{m}$ and $\mathrm{H}_{2} \mathrm{O}$ at $2.9 \mu \mathrm{m}$ and/or $10-33 \mu \mathrm{m}$ (Table 6), we also find that the depletion scenario is not limited to the known transitional disks or to disks around intermediate-mass stars. As shown in Figure 9, several disks around solar-mass stars show at least some degree of water depletion (those that we find to have $0.1 \lesssim R_{\text {co }} / R_{\text {snow }} \lesssim 1$ ). By interpreting results from Spitzer and VLT-CRIRES surveys in the context of this analysis (Figure 10 ), we could conclude that $\approx 20 \%-40 \%$ of disks around solar-mass stars may have molecular gaps in inner disks, while this fraction may be $\approx 80 \%$ for disks around intermediate-mass stars.

The analysis of velocity-resolved molecular line spectra suggests a path of disk evolution where gas-phase $\mathrm{CO}$ and water are depleted from the planet-forming region in an insideout fashion, a path that may be shared by disks around stellar masses of $0.3 \lesssim M_{\star} / M_{\odot} \lesssim 3.5$ (as probed in this sample). By detecting $\mathrm{H}_{2} \mathrm{O}$ emission only at long wavelengths in SR 9, IM Lup, HD 35929, EC 82, TW Hya, DoAr 44, and HD 163296 (the last three of this list have been previously studied by Fedele et al. 2012; Zhang et al. 2013; Salyk et al. 2015), we propose that these disks may be in an intermediate phase of depletion $\left(0.1 \lesssim R_{\text {co }} / \mathrm{R}_{\text {snow }} \lesssim 1\right)$ and still retain some water vapor in their planet-forming region. Disks that have $2.9-33 \mu \mathrm{m}$ water line fluxes lower than $\approx 5 \times 10^{-15}$ $\mathrm{erg} \mathrm{s}^{-1} \mathrm{~cm}^{-2}$ (distance $/ 140 \mathrm{pc}$ ) have $R_{\mathrm{co}} / R_{\text {snow }} \gtrsim 0.5$ and have already depleted their molecular gas in the planet-forming region within the snow line. This line flux limit may be used to identify molecular holes/gaps in inner disks when only the line flux, and not the line profile or a direct image, can be obtained.

In Figure 9 we also include for comparison the case of disks around low-mass stars $\left(M_{\star}<0.2 M_{\odot}\right)$, observed with Spitzer at 10-19 $\mu \mathrm{m}$ (Pascucci et al. 2013), divided by 10 to re-scale the strong emission observed from organic molecules (HCN and $\mathrm{C}_{2} \mathrm{H}_{2}$ ). Rovibrational $\mathrm{CO}$ emission at $4.7 \mu \mathrm{m}$ has not been obtained for any of these disks so far, so we cannot include them in the T-R diagram. However, their spectra at Spitzer wavelengths could be distinguished from that of disks with large gaps by considering the emission from organics. Walsh et al. (2015) suggested that disks around low-mass stars may be too cold to produce strong water emissions. The luminosity dependence shown in Figure 1 may provide an explanation for the nondetection of water in these disks with Spitzer (only a tentative detection was reported by Pascucci et al. 2013): models produce fluxes at or below the measured sensitivity limits, due to a radial extent of the warm water-emitting layer that is $\sim 10$ times smaller than in a disk around a solar-mass star $(\sim 0.1 \mathrm{au}$; see Walsh et al. 2015). However, according to models, these disks would still show prominent organic emission lines from a disk atmosphere at 0.1-10 au, only mildly UV-irradiated as compared to higher-mass stars. Therefore, a continuous disk around low-mass stars would produce emission at least from organics at $13-15 \mu \mathrm{m}$, while no molecular emission at $10-19 \mu \mathrm{m}$ may be the signature of inner disk depletion, with $R_{\text {co }} / R_{\text {snow }}>0.1$ also in disks around lowmass stars.

\subsection{Origin of Molecular Holes/Gaps in Inner Disks}

Previous studies proposed the idea that UV radiation may be responsible for the depletion of water vapor in inner disk surfaces, through an efficient photodissociation of $\mathrm{H}_{2} \mathrm{O}$ into $\mathrm{OH}$ (Mandell et al. 2008; Pontoppidan et al. 2010a; Fedele et al. 2011; Brittain et al. 2016). Current thermochemical disk models, however, show that the balance at thermochemical equilibrium between the production and destruction of the water molecules produces large columns of water vapor, even in highly UV-irradiated disk surfaces (e.g., Woitke et al. 2009; Walsh et al. 2015). In fact, models produce more water emission than in less UV-luminous stars (or less massive stars; see Figure 1), because $\mathrm{H}_{2} \mathrm{O}$ and $\mathrm{OH}$ are able to self-shield to 
UV radiation at the column densities typically measured in inner disks ( $\gtrsim 10^{17} \mathrm{~cm}^{-2}$; Bethell \& Bergin 2009). Therefore it is still unclear whether UV photodissociation alone may be responsible for a global depletion of water vapor in inner disks. Further, $\mathrm{CO}$ and $\mathrm{H}_{2} \mathrm{O}$ emission at 2.9-4.7 $\mu \mathrm{m}$ recede at larger disk radii together (Section 3 ), and the $\mathrm{CO}$ molecule is able to self-shield to UV photodissociation at even lower columns $\left(\approx 10^{15} \mathrm{~cm}^{-2}\right.$; Bruderer 2013). As a consequence, a depletion of the molecular column densities to below the self-shielding limits may be required before UV photodissociation may deplete any residual $\mathrm{CO}$ and $\mathrm{H}_{2} \mathrm{O}$ gas.

The destruction of $\mathrm{H}_{2} \mathrm{O}$ by $\mathrm{UV}$ radiation has been observationally tested by measuring if $\mathrm{OH}$ emission is consistent with prompt emission from excited $\mathrm{OH}$, produced by photodissociation of $\mathrm{H}_{2} \mathrm{O}$ (Carr \& Najita 2014). However, $\mathrm{H}_{2} \mathrm{O}$ line fluxes increase (instead of decrease) with increasing UV radiation, by considering the accretion luminosity as a proxy (at least up to moderate accretion luminosities; see Banzatti et al. 2012, 2014, and Figure 21). The measured $v 2 / v 1$ vibrational ratios of $\mathrm{CO}$ lines, which are sensitive to UV pumping and to the UV irradiation that reaches the disk (e.g., Thi et al. 2013), provide another way to test if and how $\mathrm{H}_{2} \mathrm{O}$ line fluxes are linked to UV irradiation. The ratio $v 2 / v 1$ decreases as water emission decreases from shorter to longer wavelengths, and $v 2 / v 1$ is not anomalously high in several disks around intermediate-mass stars that show no water emission (Figure 9). However, the increase in $v 2 / v 1$ with inner gap size for $R_{\text {co }} \gtrsim 2$ au demonstrates that enough UV radiation is present to pump the $\mathrm{CO}$ at large radial distances. In this regime of disk gaps around intermediate-mass stars, UV photodissociation of water could contribute more significantly to the depletion of residual water vapor. We find that this regime is activated after the inner disk region at $\lesssim 2$ au has already been depleted from $\mathrm{CO}$ and water.

If not through UV photodissociation, the depletion of $\mathrm{CO}$ and $\mathrm{H}_{2} \mathrm{O}$ may be initiated in inner disks by direct removal of disk gas. Disk photoevaporation by X-ray and UV photons potentially provides an inside-out dispersal mechanism, although this is proposed to be efficient only beyond the radius where the gas is gravitationally bound to the star ( $\gtrsim 1-2$ au in disks around solarmass stars). Another removal mechanism could be through portions of MHD winds (e.g., Ferreira et al. 2006; Bai 2016) that may be probed at optical wavelengths through a low-velocity component in the forbidden oxygen lines (Simon et al. 2016). A slow disk wind has been proposed to contribute also to the $\mathrm{CO}$ narrow component in double-component disks (Bast et al. 2011; Pontoppidan et al. 2011a), and may play a role in the depletion of molecular gas in inner disks. A promising direction for future work is to test if wind models can reproduce the formation and development of the molecular holes revealed by the analysis of $\mathrm{CO}$ and $\mathrm{H}_{2} \mathrm{O}$ velocity-resolved infrared spectroscopy.

\subsection{Molecular Composition and Evolution of Inner Disks}

In Figure 8, we showed linear trends in the $\mathrm{CO} / \mathrm{H}_{2} \mathrm{O}$ and $\mathrm{OH} / \mathrm{H}_{2} \mathrm{O}$ line flux ratios, as measured in the $2.9-4.7 \mu \mathrm{m}$ lines observed with CRIRES. Both ratios were characterized only out to $\log R_{\text {co }} / R_{\text {snow }} \sim-1$, due to the absence of $2.93 \mu \mathrm{m} \mathrm{H}_{2} \mathrm{O}$ detections at larger radii. To explore these trends over a larger disk region, we include in Figure 11 the line flux ratios as measured using $\mathrm{H}_{2} \mathrm{O}$ line detections at longer wavelengths from the Spitzer data set, which is sensitive to emission at larger disk radii (Sections 3 and 4). We find that the $\mathrm{CO} / \mathrm{H}_{2} \mathrm{O}$ line flux ratio of all three water lines show similar radial trends. The increase in $\mathrm{CO} / \mathrm{H}_{2} \mathrm{O}$ is confirmed out to $\log$
$R_{\text {co }} / R_{\text {snow }} \sim-0.7$ (Figure 11, left plot) by all three water lines. At larger radii, the Spitzer lines suggest a decreasing $\mathrm{CO} / \mathrm{H}_{2} \mathrm{O}$ out to the snow line radius. Beyond the snow line, lower limits show that the ratio may increase again.

Following a similar procedure, we show the measured $\mathrm{OH} /$ $\mathrm{H}_{2} \mathrm{O}$ line flux ratios in Figure 11, right plot. The constant $\sim 0.4$ ratio with disk radius is also confirmed in this case by all three $\mathrm{OH} / \mathrm{H}_{2} \mathrm{O}$ ratios as measured at different wavelengths $(2.93 \mu \mathrm{m}$ using the CRIRES data, 12.5-12.6 $\mu \mathrm{m}$ and 30-33 $\mu \mathrm{m}$ using the Spitzer data). Some disks show a higher $\mathrm{OH} / \mathrm{H}_{2} \mathrm{O}$ ratio of $\sim 1.7$ at $12.5-33 \mu \mathrm{m}$, perhaps linked to phases of stronger production of $\mathrm{OH}$ through increased UV irradiation (among these disks are, in fact, EX Lupi and DG Tau, where enhanced production of $\mathrm{OH}$ through UV photodissociation has been found by Banzatti et al. 2012 and Carr \& Najita 2014). A decreasing trend of $\mathrm{OH} / \mathrm{H}_{2} \mathrm{O}$ may be present at $\log R_{\mathrm{co}} / R_{\text {snow }} \gtrsim-0.7$. Beyond the snow line, lower limits suggest that the ratio may increase. Models propose that the $\mathrm{OH} / \mathrm{H}_{2} \mathrm{O}$ ratio in the observable column density of gas should increase significantly at the snow line, due to a drop in the column density of $\mathrm{H}_{2} \mathrm{O}$ (e.g., Walsh et al. 2015). Future velocity-resolved observations must test if the $12.5-33 \mu \mathrm{m}$ water lines have velocity profiles that match the $12.6-30 \mu \mathrm{m} \mathrm{OH}$ lines, indicating a similar emitting region (so far, no $\mathrm{OH}$ lines have been velocityresolved at wavelengths $\gtrsim 10 \mu \mathrm{m}$ ).

An open question is if these observed trends are due to the disk physical/chemical radial structure in a static "primordial" disk, rather than by the formation and evolution of disk gaps that may change it. The decrease of $\mathrm{CO} / \mathrm{H}_{2} \mathrm{O}$ and $\mathrm{OH} / \mathrm{H}_{2} \mathrm{O}$ at $\log R_{\mathrm{co}} / R_{\text {snow }} \gtrsim-0.7$ out to the snow line radius may be due to changes in the disk chemistry as molecular holes expand out to the snow line radius. A promising direction for future modeling is to calibrate the disk chemistry with the measured molecular line flux ratios. The explorations suggested previously within the framework of models that include disk dispersal processes may provide important constraints on the evolution of planet-forming regions. Interesting in this context is, for instance, the notable discrepancy between the data and the model shown in Figure 8 (panel (b) and (c)), where Antonellini et al. (2016) predict an increase in water emission when disk gaps reach the snow line radius as due to a new reservoir of water emission released by photodesorption of icy grains (I. Kamp 2016, private communication). The fact that this is not observed in the data may suggest that the desorption efficiency is overestimated, either by the adopted desorption rates or because dust grains are larger (i.e., the total surface available for ice desorption is smaller) or are removed from disk surfaces. In the evolved disk of TW Hya, to reproduce weak water lines observed at $>250 \mu \mathrm{m}$ with Herschel, Hogerheijde et al. (2011) proposed that icy dust grains may have been removed from the disk surface by settling toward the midplane, hiding a large reservoir of icy solids in a region shielded from UV irradiation and photodesorption.

\section{SUMMARY AND CONCLUSIONS}

(1) This analysis combines a sample of 55 protoplanetary disks that have $\mathrm{CO}$ rovibrational emission spectra observed at $4.7 \mu \mathrm{m}$, as well as $\mathrm{H}_{2} \mathrm{O}$ and $\mathrm{OH}$ emission spectra observed at 2.9 and/or $10-33 \mu \mathrm{m}$. $\mathrm{H}_{2} \mathrm{O}$ and $\mathrm{OH}$ spectra at $2.9 \mu \mathrm{m}$ (from VLT-CRIRES) are published here for the first time for 25 disks; $\mathrm{CO} 4.7 \mu \mathrm{m}$ spectra (from VLT-CRIRES) and $\mathrm{H}_{2} \mathrm{O}$ and $\mathrm{OH}$ spectra at $10-33 \mu \mathrm{m}$ (from Spitzer-IRS) have been 
published previously. This combined sample probes the molecular content of inner disks at radii of $\approx 0.05-20 \mathrm{au}$.

(2) The velocity-resolved line profiles of $\mathrm{H}_{2} \mathrm{O}$ and $\mathrm{OH}$ emission at $2.9 \mu \mathrm{m}$ are matched by the broader/inner component of $4.7 \mu \mathrm{m}$ CO emission as identified by Banzatti \& Pontoppidan (2015). These emission lines probe a similar inner disk region at $\approx 0.04-0.3 \mathrm{au}$, as shown by their similar profiles, and when $\mathrm{CO}$ is depleted in this region, $\mathrm{H}_{2} \mathrm{O}$ is also depleted. $\mathrm{H}_{2} \mathrm{O}$ lines at longer wavelengths $(12-33 \mu \mathrm{m})$, probing progressively colder water, may be co-spatial with the colder component of $\mathrm{CO}$ emission at disk radii of $>0.3 \mathrm{au}$.

(3) We propose the infrared water spectrum as a tracer of inside-out clearing of inner disks around stars of masses between 0.3 and $3.5 M_{\odot}$. We find a wavelength-dependent decrease of water line fluxes, where water emission is absent at $2.9 \mu \mathrm{m}$ but still present at longer wavelengths $(12-33 \mu \mathrm{m})$ in some disks. We discover a correlation between water line fluxes at $2.9-33 \mu \mathrm{m}$ and the size of inner disk gaps/holes as probed by $\mathrm{CO}$ emission, where water emission decreases as $R_{\text {co }}$ approaches the snow line radius. All taken together, we interpret this as due to the progressive depletion of inner disks in an inside-out fashion, where inner/hotter gas is depleted before outer/colder gas.

(4) This analysis helps clarify the origin of the absence of infrared $\mathrm{H}_{2} \mathrm{O}$ emission in disks around intermediate-mass stars: we find that these disks have inner gaps or holes in molecular gas (i.e., they have large $R_{\text {co }}$ ). This adds to growing evidence from other studies of gaps and holes in dust emission (e.g., Maaskant et al. 2013; Menu et al. 2015), as well as to recent model explorations (Antonellini et al. 2016).

(5) We measure the radial profiles of $\mathrm{CO} / \mathrm{H}_{2} \mathrm{O}$ and $\mathrm{OH} / \mathrm{H}_{2} \mathrm{O}$ line flux ratios in inner disks out to the water snow line. We find an increasing $\mathrm{CO} / \mathrm{H}_{2} \mathrm{O}$ with disk radius within the snow line, possibly followed by an inversion in the trend with a $\mathrm{CO} /$ $\mathrm{H}_{2} \mathrm{O}$ ratio decreasing with disk radius as the molecular hole expands out the snow line radius. For $\mathrm{OH} / \mathrm{H}_{2} \mathrm{O}$, we find a constant ratio within the snow line, possibly followed by a decrease close to the snow line.

To conclude, our work finds that the decrease in $\mathrm{CO}$ and $\mathrm{H}_{2} \mathrm{O}$ line fluxes is linked to the depletion of molecular gas through inside-out formation of inner disk gaps or holes. Line flux measurements and trends from this work provide an opportunity to model the properties of evolving inner disks and to investigate how physical changes (e.g., in the gas-to-dust ratio, dust grain growth, and the removal of inner disk gas) may affect the composition of planet-forming disk regions (e.g., through an enhanced UV penetration at large disk radii and the depletion of molecular gas from inner disks). Measured radial trends in the chemistry and depletion of water during the evolution of protoplanetary disks may offer new ground to investigate the origins of radial gradients in composition measured in Solar System bodies (e.g., Morbidelli et al. 2012) and potentially also in exoplanet populations.

\subsection{Future Prospects with the James Webb Space Telescope (JWST)}

JWST will provide $R \sim 3000$ (or $\sim 100 \mathrm{~km} \mathrm{~s}^{-1}$ ) spectroscopy of molecular spectra from the $2.9 \mu \mathrm{m}$ lines included in this analysis (with $J W S T$-NIRSpec) to the longer wavelengths covered by Spitzer (up to $28 \mu \mathrm{m}$, with JWST-MIRI). The observed molecular emission spectra will include $\mathrm{CO}, \mathrm{H}_{2} \mathrm{O}, \mathrm{OH}$, and $\mathrm{CO}_{2}$. The broad spectral coverage will provide the data to study more molecular trends than those included in this work, and to better characterize the chemistry and its evolution when gaps form in disks. JWST also has the potential to study infrared molecular emission in disks around low-mass stars, and disk samples not limited to the closest star-forming regions at $120-180$ pc. A JWST spectrum at 3-28 $\mu \mathrm{m}$ may distinguish the evolutionary phase of inner disks from the measured line fluxes and the molecular species detected (Figure 9), spanning a range of disk radii and gap sizes ( 0.05-20 au; Banzatti \& Pontoppidan 2015) that is highly complementary to the larger scales probed by millimeter interferometry with the Atacama Large Millimeter Array ALMA $\gtrsim 5$ au at 140 pc; e.g., van der Marel et al. 2016) and by direct imaging techniques. The joint use of these observatories can transform our understanding of the development of disk gaps from the innermost disk regions to the larger-scale disk structures, and of the physical and chemical evolution of planet-forming environments.

We thank S. Antonellini and I. Kamp for providing water emission spectra from their ProDiMo model explorations, and for insightful discussions on water emission in disks and on its dependence on disk model parameters. We also thank A. Carmona for providing the CRIRES spectrum of HD139614. A.B. wishes to thank all colleagues who made this work possible through their pioneering work in infrared spectroscopy of disks. A.B. thanks Simon Bruderer for the seminal work he did in 2011-2012, which provided knowledge and tools to enable this work. A.B. acknowledges financial support by a NASA Origins of the Solar System grant No. OSS 11-OSS110120, a NASA Planetary Geology and Geophysics Program under grant NAG 5-10201. E.v.D. acknowledges European Union A-ERC grant 291141 CHEMPLAN. This work is partly based on observations made with ESO telescopes at the Paranal Observatory under programs 179.C-0151, 093.C-0432, 088.C0666, 079.C-0349, 081.C-0833, 091.C-0671, 082.C-0491, and 088.C-0898. This work is partly based on observations made with the Spitzer Space Telescope, which is operated by the Jet Propulsion Laboratory, California Institute of Technology. This publication makes use of data products from the Wide-field Infrared Survey Explorer, which is a joint project of the University of California, Los Angeles, and the Jet Propulsion Laboratory/California Institute of Technology, funded by the National Aeronautics and Space Administration. This research has made use of the VizieR catalog access tool, CDS, Strasbourg, France. The original description of the VizieR service was published in A\&AS 143, 23. This work is partly based on observations obtained at the W. M. Keck Observatory, which is operated as a scientific partnership among the California Institute of Technology, the University of California, and the National Aeronautics and Space Administration. The observatory was made possible by the generous financial support of the W. M. Keck Foundation.

\section{APPENDIX}

\section{A.1. Line Flux Measurements}

We measure emission line fluxes by fitting a first-order polynomial continuum over neighboring pixels and by summing continuum-subtracted pixel flux values over the spectral range of each line. In the Spitzer-IRS spectra, to select pixels of continuum free from line emission, we use models of 
Table 6

Line Fluxes

\begin{tabular}{|c|c|c|c|c|c|c|c|c|}
\hline \multirow{2}{*}{ Name } & \multicolumn{3}{|c|}{$\mathrm{H}_{2} \mathrm{O}$} & \multicolumn{3}{|c|}{$\mathrm{OH}$} & \multicolumn{2}{|c|}{$\operatorname{CO} v=1-0 \mathrm{P} 10$} \\
\hline & $2.93 \mu \mathrm{m}$ & $12.52 \mu \mathrm{m}$ & $33 \mu \mathrm{m}$ & $2.93 \mu \mathrm{m}$ & $12.65 \mu \mathrm{m}$ & $30 \mu \mathrm{m}$ & $\mathrm{BC}$ & $\mathrm{NC}$ \\
\hline AATau & $<0.25$ & $0.61 \pm 0.04$ & $2.48 \pm 0.10$ & $0.11 \pm 0.00$ & $0.29 \pm 0.03$ & $2.01 \pm 0.08$ & $\ldots$ & $2.74 \pm 0.02$ \\
\hline AS205 N & $7.33 \pm 2.71$ & $11.26 \pm 0.61$ & $29.32 \pm 1.88$ & $2.06 \pm 0.76$ & $6.20 \pm 0.61$ & $30.84 \pm 1.34$ & $17.98 \pm 7.19$ & $13.38 \pm 5.35$ \\
\hline AS209 & $\ldots$ & $\ldots$ & $\ldots$ & $\ldots$ & $\ldots$ & $\ldots$ & $1.04 \pm 0.03$ & $0.71 \pm 0.02$ \\
\hline CVCha & $\ldots$ & $\ldots$ & $\ldots$ & $\ldots$ & $\ldots$ & $\ldots$ & $3.55 \pm 0.11$ & $1.97 \pm 0.07$ \\
\hline CWTau & $<0.46$ & $3.59 \pm 0.09$ & $8.42 \pm 0.06$ & $1.17 \pm 0.21$ & $1.54 \pm 0.07$ & $10.66 \pm 0.13$ & $5.80 \pm 0.93$ & $5.51 \pm 0.88$ \\
\hline DFTau & $4.55 \pm 0.59$ & $\ldots$ & $\ldots$ & $1.65 \pm 0.21$ & $\ldots$ & $\ldots$ & $7.99 \pm 0.72$ & $3.25 \pm 0.29$ \\
\hline DGTau & $\ldots$ & $2.49 \pm 0.20$ & $4.70 \pm 0.70$ & $\ldots$ & $5.50 \pm 0.18$ & $16.60 \pm 0.64$ & $5.42 \pm 0.43$ & $\ldots$ \\
\hline DoAr24E S & $<1.09$ & $1.66 \pm 0.15$ & $9.85 \pm 0.35$ & $1.92 \pm 0.73$ & $2.13 \pm 0.12$ & $6.44 \pm 0.34$ & $10.15 \pm 3.45$ & $5.51 \pm 1.87$ \\
\hline DoAr44 & $<2.02$ & $0.52 \pm 0.05$ & $4.39 \pm 0.16$ & $<0.56$ & $0.36 \pm 0.05$ & $3.00 \pm 0.15$ & $\ldots$ & $2.24 \pm 0.03$ \\
\hline DOTau & $2.31 \pm 0.19$ & $1.46 \pm 0.07$ & $2.63 \pm 0.33$ & $1.01 \pm 0.08$ & $2.19 \pm 0.06$ & $3.39 \pm 0.22$ & $4.44 \pm 0.27$ & $0.59 \pm 0.04$ \\
\hline DRTau & $2.84 \pm 0.43$ & $5.25 \pm 0.12$ & $10.11 \pm 0.13$ & $0.80 \pm 0.12$ & $2.37 \pm 0.13$ & $6.97 \pm 0.28$ & $8.36 \pm 1.34$ & $6.55 \pm 1.05$ \\
\hline EC82 & $<0.60$ & $<0.36$ & $2.05 \pm 0.18$ & $0.39 \pm 0.02$ & $<0.33$ & $1.73 \pm 0.17$ & $\ldots$ & $2.04 \pm 0.02$ \\
\hline EXLup08 & $8.69 \pm 2.61$ & $<0.99$ & $5.99 \pm 0.90$ & $3.88 \pm 1.16$ & $1.89 \pm 0.24$ & $3.85 \pm 1.53$ & $13.20 \pm 7.92$ & $5.51 \pm 3.31$ \\
\hline EXLup14 & $0.32 \pm 0.02$ & $1.00 \pm 0.16$ & $3.43 \pm 0.48$ & $0.12 \pm 0.01$ & $<0.46$ & $<1.14$ & $0.70 \pm 0.02$ & $0.89 \pm 0.03$ \\
\hline FNTau & $<0.05$ & $<0.19$ & $<0.27$ & $<0.03$ & $0.21 \pm 0.05$ & $<0.42$ & $\ldots$ & $0.29 \pm 0.01$ \\
\hline FZTau & $\cdots$ & $5.94 \pm 0.10$ & $6.44 \pm 0.05$ & $\ldots$ & $1.67 \pm 0.08$ & $5.38 \pm 0.11$ & $\ldots$ & $9.59 \pm 0.68$ \\
\hline GQLup & $<0.57$ & $0.70 \pm 0.04$ & $4.92 \pm 0.09$ & $<0.26$ & $0.51 \pm 0.04$ & $3.06 \pm 0.11$ & $4.18 \pm 0.21$ & $2.22 \pm 0.12$ \\
\hline HD36112 & $\ldots$ & $<1.85$ & $<6.55$ & $\ldots$ & $<1.81$ & $9.76 \pm 1.36$ & $\ldots$ & $8.85 \pm 3.45$ \\
\hline HD95881* & $\ldots$ & $<2.24$ & $<3.00$ & $\ldots$ & $<2.19$ & $<2.03$ & $\ldots$ & $4.91 \pm 0.20$ \\
\hline HD97048* & $\ldots$ & $\ldots$ & $\ldots$ & $\ldots$ & $\ldots$ & $\ldots$ & $\ldots$ & $12.30 \pm 0.40$ \\
\hline HD98922* & $<0.08$ & $<7.48$ & $<6.05$ & $<0.08$ & $<7.29$ & $7.29 \pm 2.66$ & $\ldots$ & $5.08 \pm 0.14$ \\
\hline HD101412 & $\ldots$ & $<1.17$ & $<1.27$ & $\ldots$ & $<1.14$ & $1.89 \pm 0.25$ & $\ldots$ & $\ldots$ \\
\hline HD135344B & $<0.30$ & $<0.65$ & $<1.84$ & $<0.14$ & $<0.63$ & $3.66 \pm 0.36$ & $\cdots$ & $1.99 \pm 0.79$ \\
\hline HD139614 & $\ldots$ & $\ldots$ & $\ldots$ & $\ldots$ & $\ldots$ & $\ldots$ & $\ldots$ & $1.36 \pm 0.05$ \\
\hline HD141569* & $\cdots$ & $\ldots$ & $\ldots$ & $\cdots$ & $\ldots$ & $\ldots$ & $\cdots$ & $1.77 \pm 0.14$ \\
\hline HD142527 & $\ldots$ & $<2.37$ & $<10.41$ & $\ldots$ & $<2.31$ & $14.30 \pm 2.00$ & $\ldots$ & $8.58 \pm 4.29$ \\
\hline HD144432S & $\cdots$ & $\ldots$ & $\ldots$ & $\cdots$ & $\ldots$ & $\ldots$ & $\cdots$ & $2.73 \pm 0.71$ \\
\hline HD150193* & $\ldots$ & $<3.55$ & $<7.00$ & $\ldots$ & $<3.46$ & $10.40 \pm 0.94$ & $\ldots$ & $8.22 \pm 1.94$ \\
\hline HD163296* & $\cdots$ & $<3.91$ & $7.21 \pm 1.02$ & $\cdots$ & $<3.83$ & $24.77 \pm 0.99$ & $\cdots$ & $18.70 \pm 1.60$ \\
\hline HD179218* & $\cdots$ & $<5.27$ & $<9.89$ & $\cdots$ & $<4.50$ & $<4.89$ & $\cdots$ & $2.88 \pm 0.74$ \\
\hline HD190073* & $\ldots$ & $<2.43$ & $<1.18$ & $\ldots$ & $<2.38$ & $5.77 \pm 0.59$ & $\ldots$ & $6.99 \pm 0.59$ \\
\hline HD244604 & $\ldots$ & $<1.53$ & $<1.66$ & $\ldots$ & $<1.50$ & $<0.95$ & $\ldots$ & $0.64 \pm 0.01$ \\
\hline HD250550* & $<0.14$ & $\ldots$ & $\ldots$ & $0.14 \pm 0.01$ & $\ldots$ & $\ldots$ & $\ldots$ & $5.80 \pm 0.35$ \\
\hline HTLup & $<0.73$ & $<1.15$ & $<1.19$ & $0.36 \pm 0.06$ & $1.26 \pm 0.31$ & $2.58 \pm 0.48$ & $2.27 \pm 0.25$ & $0.49 \pm 0.06$ \\
\hline IMLup & $<0.18$ & $<0.18$ & $0.65 \pm 0.12$ & $<0.08$ & $0.34 \pm 0.04$ & $<0.34$ & $0.44 \pm 0.01$ & $0.07 \pm 0.11$ \\
\hline IRS48 & $\ldots$ & $\ldots$ & $\ldots$ & $\ldots$ & $\ldots$ & $\ldots$ & $\ldots$ & $0.58 \pm 0.13$ \\
\hline LkHa330 & $\ldots$ & $<0.23$ & $<1.77$ & $\ldots$ & $<0.23$ & $<0.82$ & $\ldots$ & $1.01 \pm 0.09$ \\
\hline RNO90 & $2.61 \pm 0.34$ & $7.38 \pm 0.26$ & $23.29 \pm 0.35$ & $1.40 \pm 0.18$ & $2.98 \pm 0.25$ & $19.66 \pm 0.35$ & $12.93 \pm 2.07$ & $6.24 \pm 1.00$ \\
\hline RULup & $4.27 \pm 0.60$ & $3.08 \pm 0.16$ & $5.00 \pm 0.23$ & $1.60 \pm 0.22$ & $6.08 \pm 0.16$ & $7.39 \pm 0.21$ & $6.63 \pm 0.73$ & $4.93 \pm 0.54$ \\
\hline RYLup & $\ldots$ & $<0.96$ & $<1.52$ & $\ldots$ & $<0.93$ & $2.11 \pm 0.30$ & $\ldots$ & $1.76 \pm 0.09$ \\
\hline RWAur & $\ldots$ & $6.79 \pm 0.20$ & $9.48 \pm 0.17$ & $\ldots$ & $2.09 \pm 0.17$ & $8.35 \pm 0.25$ & $8.47 \pm 0.38$ & $5.83 \pm 0.25$ \\
\hline SCrA S & $6.46 \pm 2.00$ & $8.59 \pm 0.87$ & $23.16 \pm 2.33$ & $3.85 \pm 1.19$ & $13.32 \pm 0.82$ & $40.11 \pm 1.69$ & $13.25 \pm 2.92$ & $4.86 \pm 1.07$ \\
\hline SCrA N & $8.68 \pm 2.69$ & $8.59 \pm 0.87$ & $23.16 \pm 2.33$ & $3.75 \pm 1.16$ & $13.32 \pm 0.82$ & $40.11 \pm 1.69$ & $8.11 \pm 1.78$ & $5.76 \pm 1.27$ \\
\hline SR9 & $\ldots$ & $<0.93$ & $2.42 \pm 0.21$ & $\ldots$ & $<0.91$ & $3.27 \pm 0.13$ & $\ldots$ & $1.31 \pm 0.03$ \\
\hline SR21 & $<0.03$ & $<1.16$ & $<4.60$ & $<0.02$ & $<1.13$ & $14.42 \pm 0.98$ & $\ldots$ & $0.46 \pm 0.02$ \\
\hline TTau N & $12.77 \pm 10.22$ & $\ldots$ & $\ldots$ & $6.14 \pm 4.91$ & $\ldots$ & $\ldots$ & $32.12 \pm 25.70$ & $9.65 \pm 7.72$ \\
\hline TTau S & $9.66 \pm 7.73$ & $\ldots$ & $\ldots$ & $11.31 \pm 9.05$ & $\ldots$ & $\ldots$ & $37.56 \pm 30.05$ & $\ldots$ \\
\hline TWCha & $0.49 \pm 0.00$ & $0.62 \pm 0.02$ & $2.42 \pm 0.05$ & $0.10 \pm 0.00$ & $0.19 \pm 0.02$ & $1.41 \pm 0.04$ & $\ldots$ & $1.05 \pm 0.01$ \\
\hline TWHya & $<0.02$ & $0.20 \pm 0.05$ & $3.96 \pm 0.47$ & $<0.01$ & $0.58 \pm 0.04$ & $10.21 \pm 0.40$ & $\ldots$ & $0.64 \pm 0.00$ \\
\hline VSSG1 & $\cdots$ & $2.11 \pm 0.26$ & $7.49 \pm 0.45$ & $\ldots$ & $1.20 \pm 0.24$ & $4.94 \pm 0.35$ & $4.83 \pm 0.29$ & $2.88 \pm 0.17$ \\
\hline VVSer & $\ldots$ & $<1.27$ & $<1.73$ & $\ldots$ & $1.88 \pm 0.32$ & $4.48 \pm 0.25$ & $\ldots$ & $2.02 \pm 0.36$ \\
\hline VWCha & $2.07 \pm 0.11$ & $1.97 \pm 0.08$ & $7.14 \pm 0.08$ & $1.32 \pm 0.07$ & $1.38 \pm 0.07$ & $9.85 \pm 0.12$ & $6.46 \pm 0.30$ & $2.96 \pm 0.14$ \\
\hline VZCha & $0.74 \pm 0.01$ & $0.83 \pm 0.05$ & $2.46 \pm 0.07$ & $0.30 \pm 0.00$ & $0.52 \pm 0.05$ & $1.97 \pm 0.05$ & $3.11 \pm 0.03$ & $1.01 \pm 0.02$ \\
\hline WaOph6 & $2.66 \pm 0.19$ & $1.29 \pm 0.04$ & $2.56 \pm 0.14$ & $1.32 \pm 0.09$ & $1.00 \pm 0.04$ & $3.30 \pm 0.12$ & $2.75 \pm 0.14$ & $0.96 \pm 0.05$ \\
\hline WXCha & $<0.51$ & $1.18 \pm 0.04$ & $3.08 \pm 0.07$ & $0.32 \pm 0.01$ & $0.39 \pm 0.04$ & $1.63 \pm 0.06$ & $1.98 \pm 0.03$ & $0.66 \pm 0.02$ \\
\hline
\end{tabular}

Note. Line fluxes are reported in $10^{-14} \mathrm{erg} \mathrm{s}^{-1} \mathrm{~cm}^{-2}$, equivalent to $10^{-17} \mathrm{~W} \mathrm{~m}^{-2}$. Upper limits are given as $2 \times \sigma$, where $\sigma$ is the sum of pixel flux errors. For targets marked with *, the CO line flux is adopted from van der Plas et al. (2015), Hein Bertelsen et al. (2016), and Brittain et al. (2007), while the $\mathrm{H}_{2} \mathrm{O}$ and $\mathrm{OH}$ line fluxes at $2.9 \mu \mathrm{m}$, where available, are adopted from Fedele et al. (2011).

the strongest emitters at $10-35 \mu \mathrm{m}$, as identified in previous work (water, $\mathrm{OH}, \mathrm{HCN}, \mathrm{C}_{2} \mathrm{H}_{2}, \mathrm{CO}_{2}$, $\mathrm{HI}$; see, e.g., Carr \& Najita 2011; Banzatti et al. 2012). The following are the ranges adopted: the $12.52 \mu \mathrm{m}$ line is measured on $12.50-12.537 \mu \mathrm{m}$, and its continuum fitted on 12.48-12.5 $\mu \mathrm{m}$ and $12.7-12.72 \mu \mathrm{m}$ (at the two sides of the line); the $33 \mu \mathrm{m}$ line on 32.92-33.06 $\mu \mathrm{m}$ 

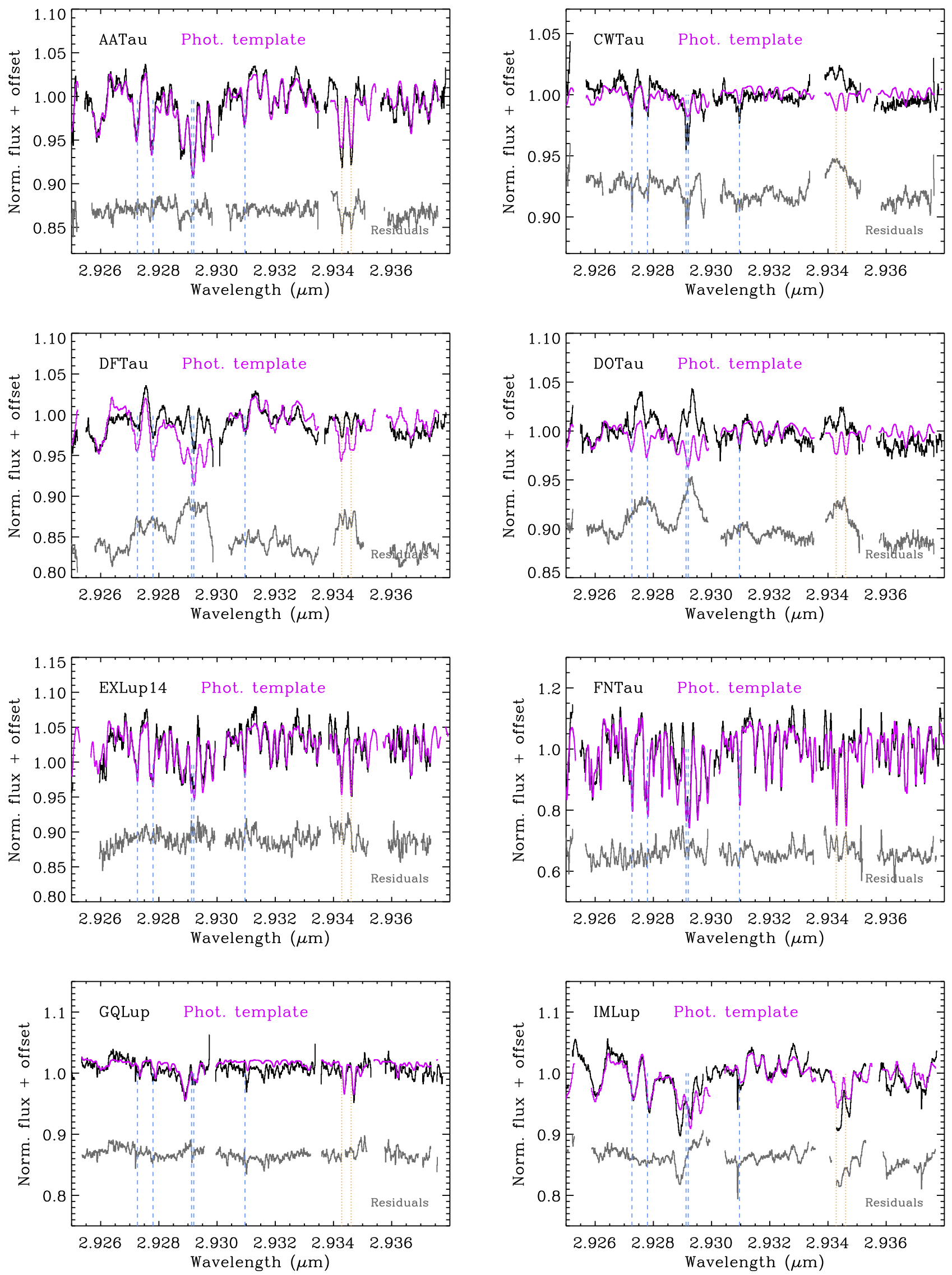

Figure 12. Photospheric correction of $2.9 \mu \mathrm{m}$ disk emission spectra observed by VLT-CRIRES. 

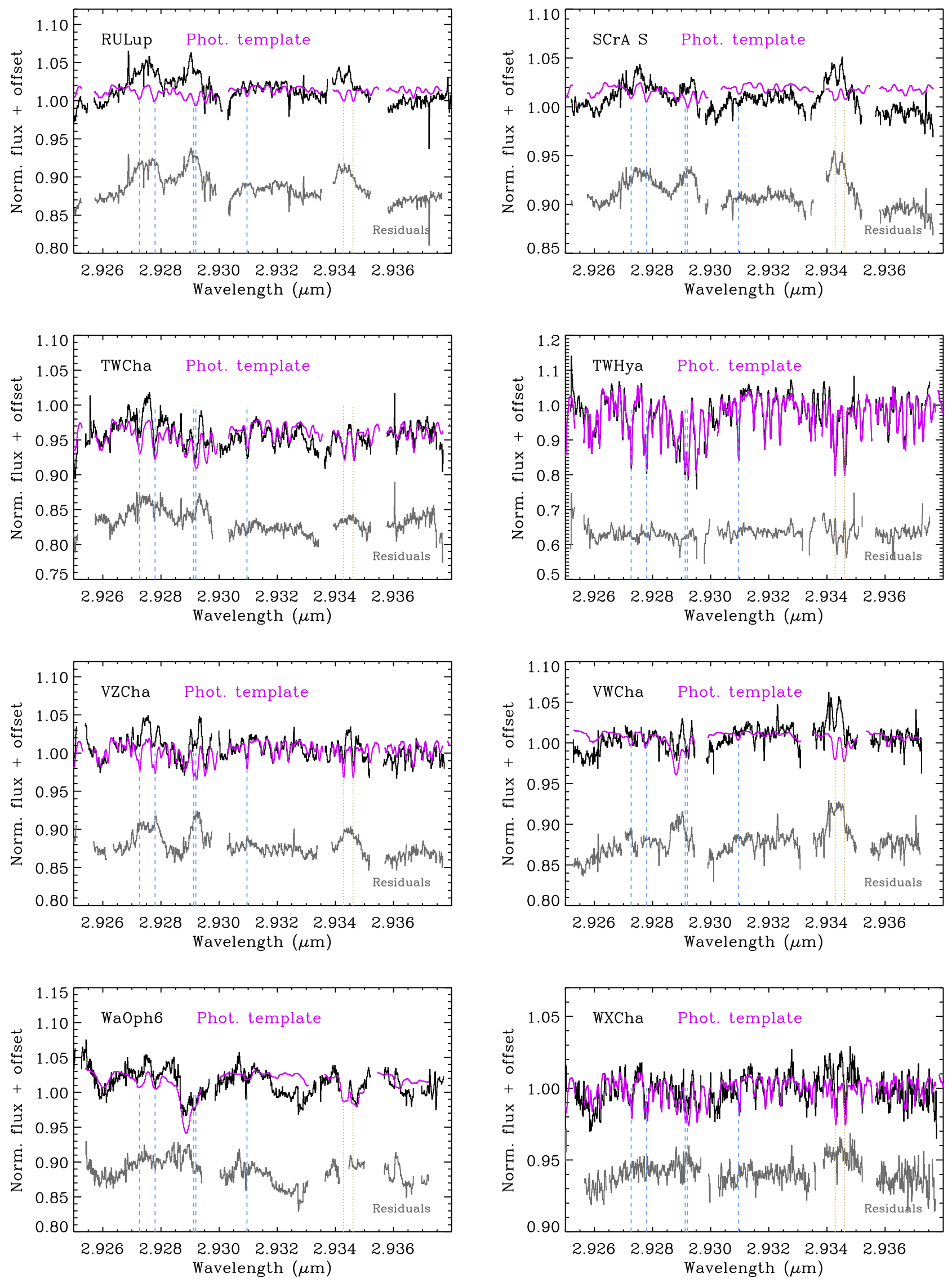

Figure 13. (Continued) Photospheric correction of $2.9 \mu \mathrm{m}$ disk emission spectra observed by VLT-CRIRES. 

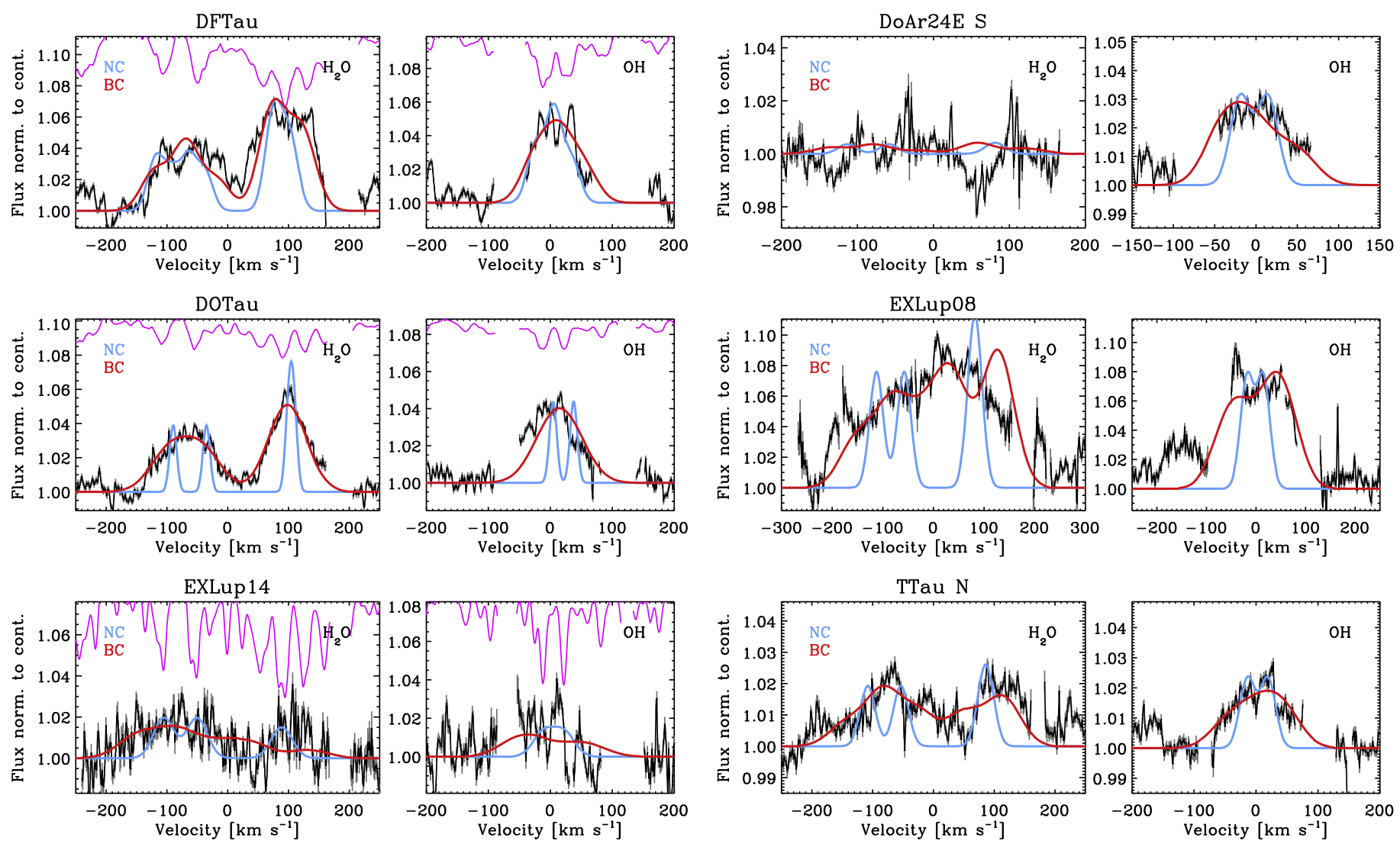

TTau S
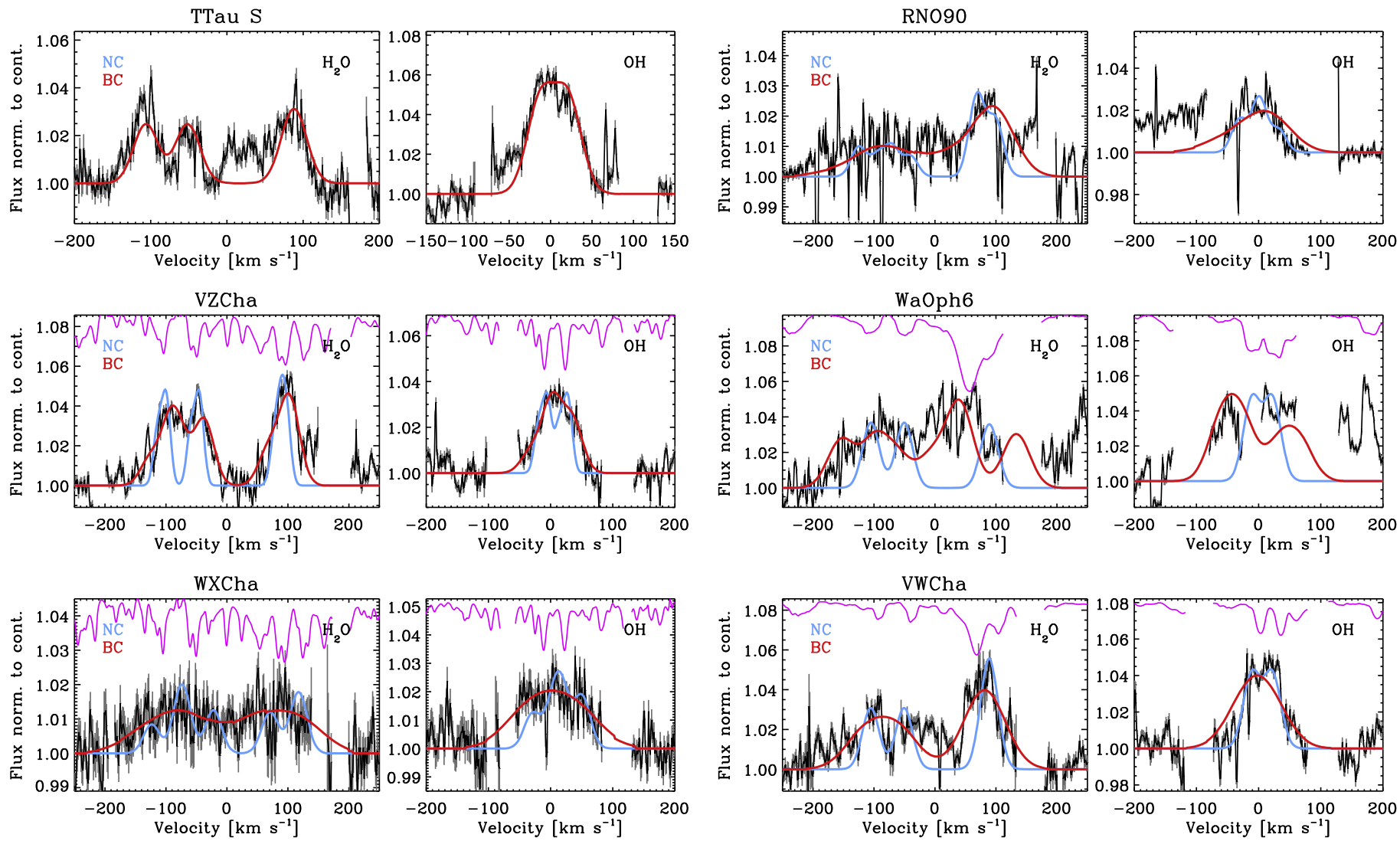

Figure 14. Fits to $\mathrm{H}_{2} \mathrm{O}$ and $\mathrm{OH}$ line profiles at $2.9 \mu \mathrm{m}$ by using the $\mathrm{BC}$ (red) and $\mathrm{NC}$ (cyan) profiles from CO emission. Spectra where photospheric correction has been applied are shown with the photospheric template in magenta at the top, broadened and veiled. The NC in TTau S is not available due to a central broad absorption component (see Brown et al. 2013). 

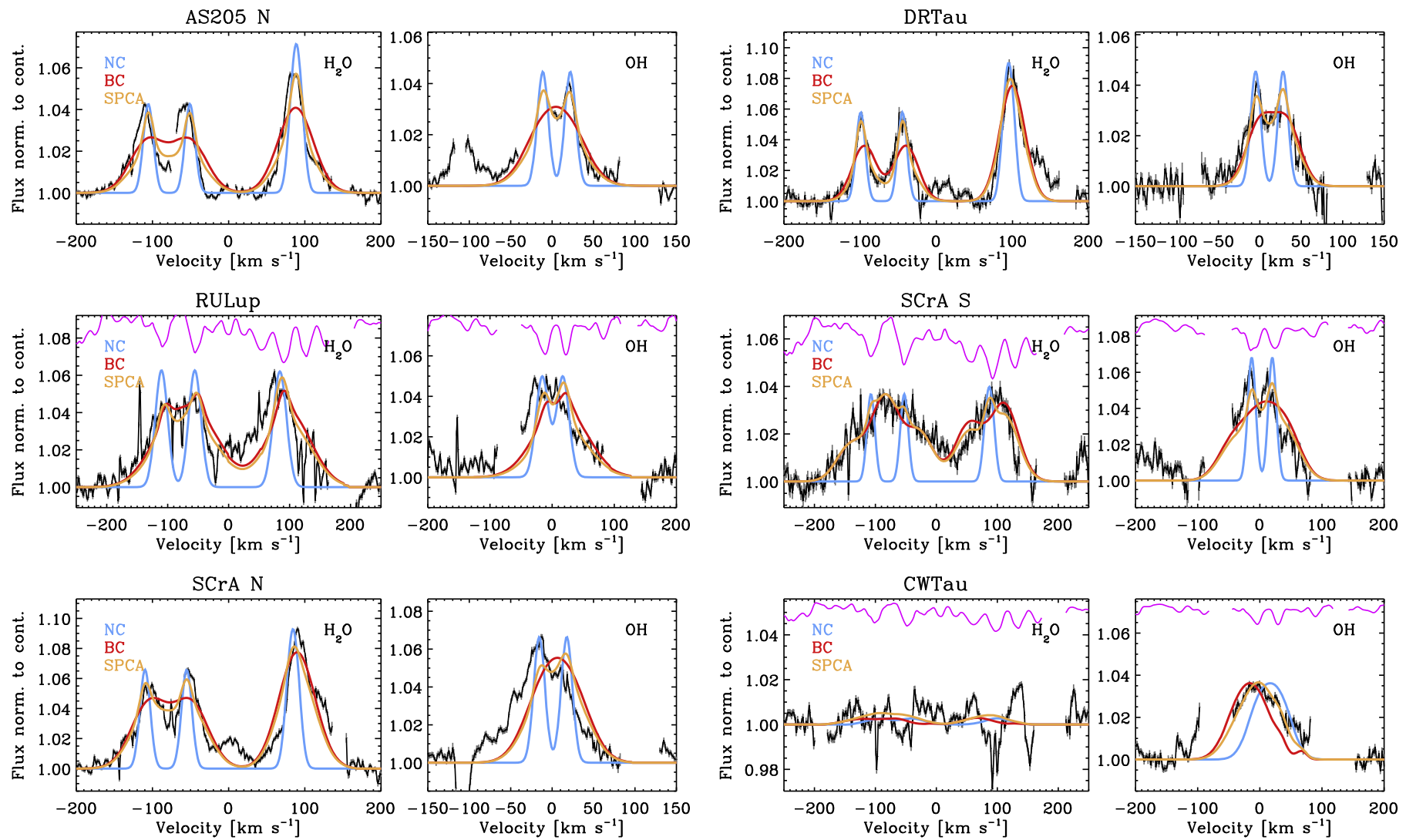

Figure 15. $\mathrm{H}_{2} \mathrm{O}$ and $\mathrm{OH}$ lines at $2.9 \mu$ m where a weak $\mathrm{NC}$ is detected on top of $\mathrm{BC}$ in $\mathrm{H}_{2} \mathrm{O}$ and/or $\mathrm{OH}$ emission. In the spectrum of $\mathrm{CW}$ Tau, only $\mathrm{OH}$ is detected. The detection of NC is only tentative in the case of RU Lup and SCrA S, due to possible photospheric residuals. The photospheric template, where used for photospheric correction, is shown in magenta at the top, broadened and veiled. Line fits follow the same color code as in Figure 14, and the SPCA fit is shown in orange (see Section A.3).
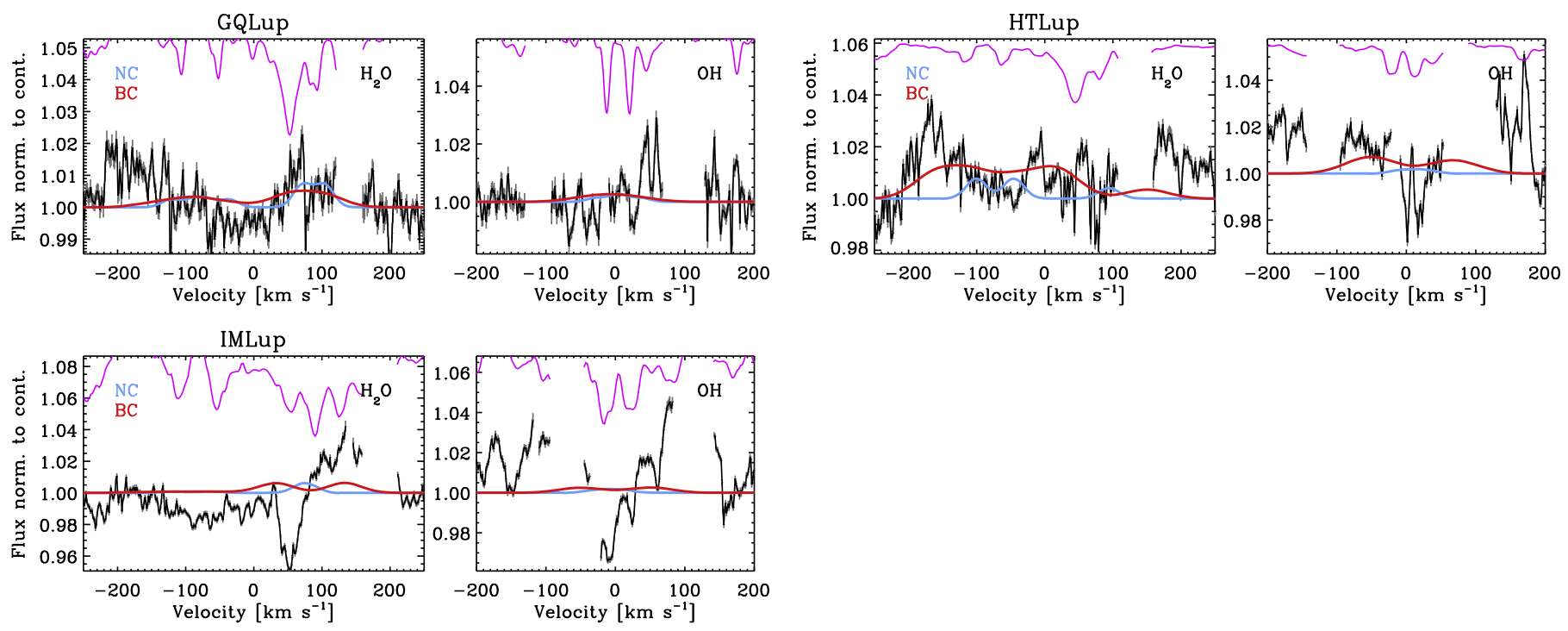

Figure 16. $2.9 \mu \mathrm{m}$ spectra with photospheric residuals due to ambiguous/inappropriate match with the spectral type of the photospheric template. The photospheric template is shown in magenta at the top, broadened and veiled. Line fits follow the same color code as in Figure 14.

and its continuum on $32.53-32.63 \mu \mathrm{m}$ and $33.4-33.47 \mu \mathrm{m}$; the $12.65 \mu \mathrm{m} \mathrm{OH}$ line on $12.64-12.68 \mu \mathrm{m}$ and its continuum on $12.48-12.5 \mu \mathrm{m}$ and $12.7-12.72 \mu \mathrm{m}$; the $30 \mu \mathrm{m} \mathrm{OH}$ lines on $30.245-30.385 \mu \mathrm{m}$ and $30.62-30.73 \mu \mathrm{m}$, and their continua on $30.1-30.17 \mu \mathrm{m}$ and $30.38-30.43 \mu \mathrm{m}$, and $30.57-30.62 \mu \mathrm{m}$ and $30.73-30.83 \mu \mathrm{m}$ (we sum the flux of these $\mathrm{OH}$ doublets to increase the $\mathrm{S} / \mathrm{N}$ ). $\mathrm{OH}$ detections at $30 \mu \mathrm{m}$ are only tentative in some spectra of disks around intermediate-mass stars and will need to be confirmed by higher-spectral-resolution observations. Line flux errors are estimated as the standard deviation of the distribution of measured line fluxes after re-sampling the spectrum using the local noise, as in Banzatti et al. (2012). Following this procedure, we find values consistent within $1 \sigma$ (or a few \%) to those measured by Pontoppidan et al. (2010a), adopting as a test case the $17.22 \mu \mathrm{m}$ water line. Line fluxes and errors are reported in Table 6; in all figures throughout this 

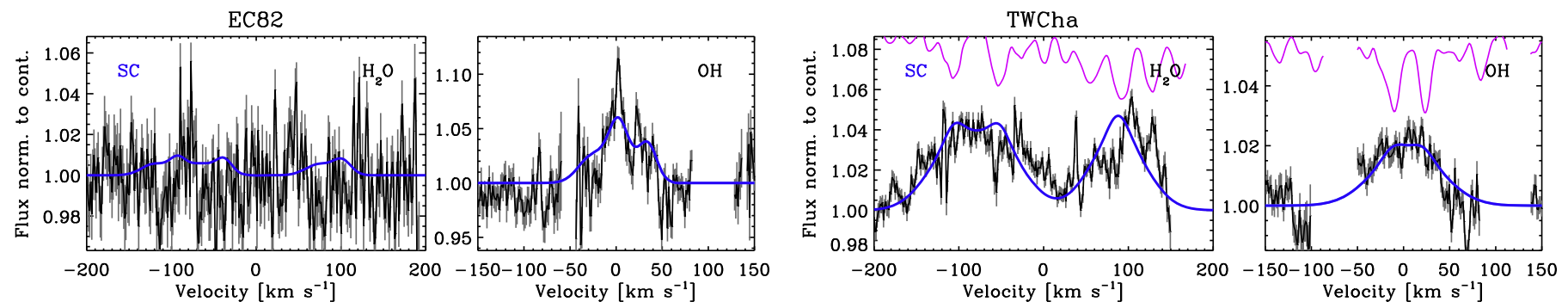

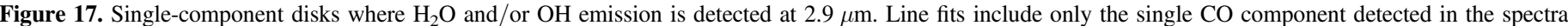
(SC). The photospheric template, where used for photospheric correction, is shown in magenta at the top, broadened and veiled.
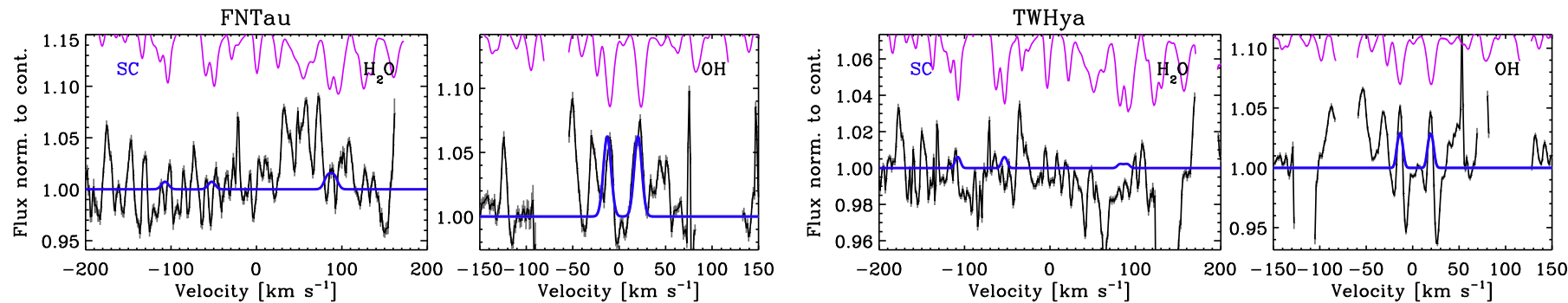

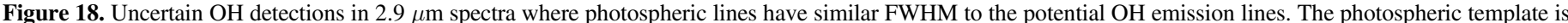

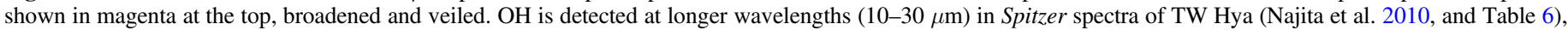
and tentatively also in FN Tau.
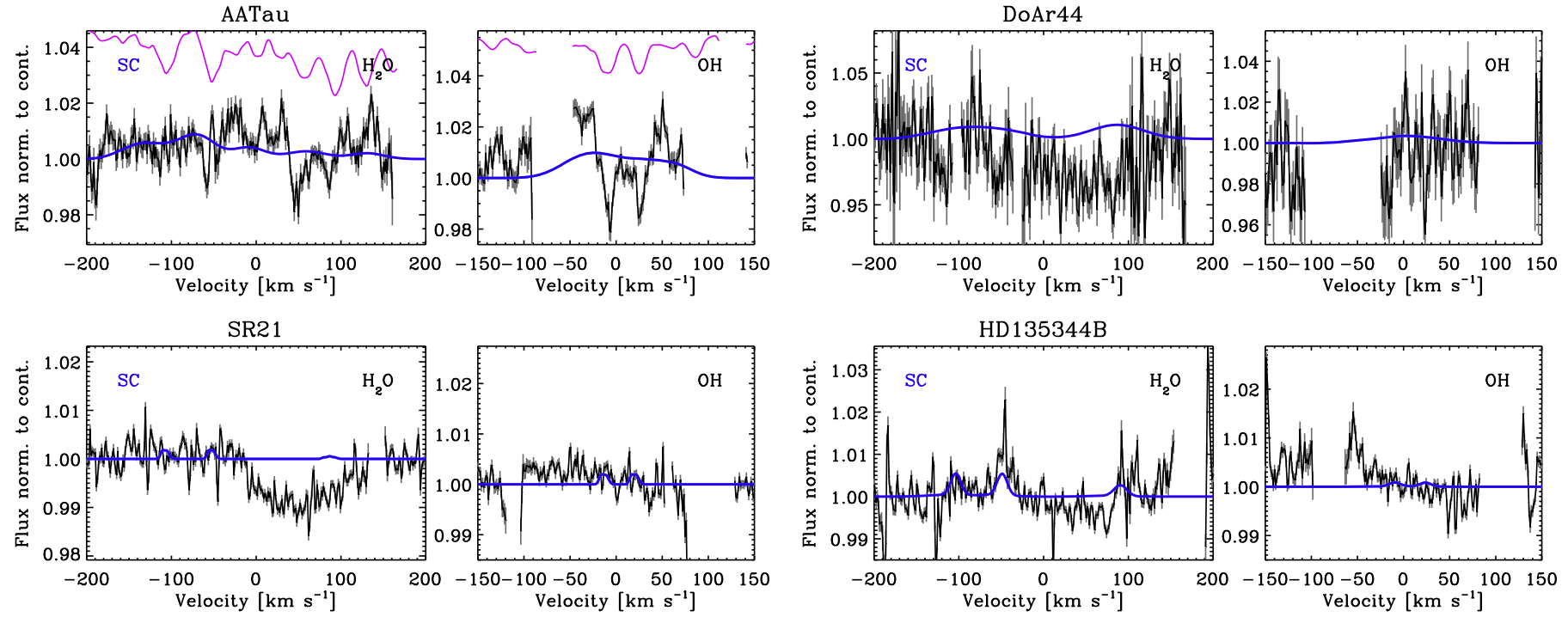

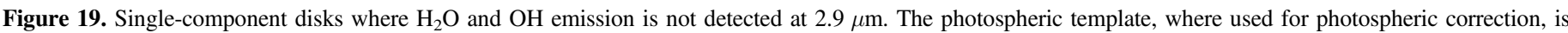
shown in magenta at the top, broadened and veiled.

paper, line fluxes are normalized to a common distance of $140 \mathrm{pc}$ for a homogeneous comparison, using distances from Table 2.

In the CRIRES spectra, line fluxes are measured in the continuum-normalized spectra and then flux calibrated using fluxes measured by the Wide-field Infrared Survey Explorer (WISE, Wright et al. 2010) at 3.35 and $4.6 \mu \mathrm{m}$, as released by Cutri et al. (2012, 2014). In some cases where the WISE $4.6 \mu \mathrm{m}$ measurement disagrees with previous photometry, we adopt the Spitzer-IRAC measurement or an interpolated value between WISE measurements at $3.35 \mu \mathrm{m}$ and $11.6 \mu \mathrm{m}$. The adopted continuum fluxes are reported in Table 2. Unlike in the Spitzer spectra, the lines observed with CRIRES are velocity-resolved and present a broad range of widths that makes it inappropriate to adopt fixed pixel ranges for their measurement. To adopt a homogeneous method, we therefore measure the line flux included within the full width at $10 \%$ of the line peak. Table 6 reports the fluxes and errors measured for the lines listed in Table 3. The four water lines at $\sim 2.93 \mu \mathrm{m}$ are measured together into a single flux value, because in most spectra they are blended together due to their large line widths.

\section{A.2. Photospheric Correction Plots}

Figures 12 and 13 show the stellar photospheric correction of CRIRES $2.9 \mu \mathrm{m}$ spectra as explained in Section 3.1. This procedure proves to be effective in correcting most science 

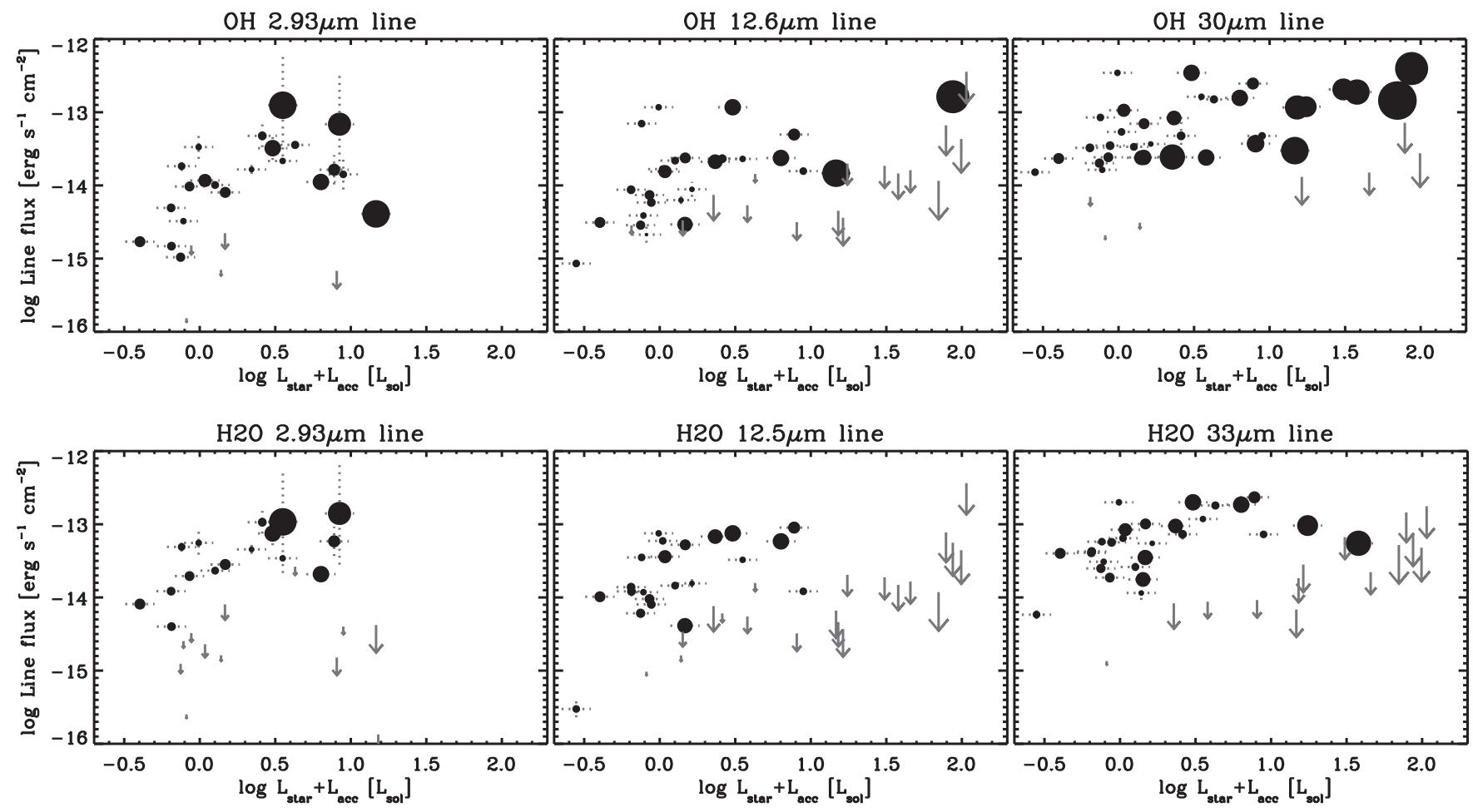

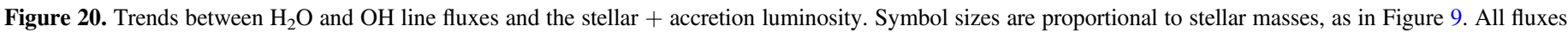
are normalized to a common distance of $140 \mathrm{pc}$.

spectra-those that match well with a spectral template and where the disk gas emission lines are broader than the absorption lines from the same molecules in the stellar photosphere. In some cases, however, there is no unambiguous/acceptable spectral match with a template (in CW Tau, HT Lup, and IM Lup), or the disk emission lines are possibly as narrow as the photospheric lines (in FN Tau and TW Hya); in these cases, the residuals after photospheric correction leave larger uncertainties on potential detections of $\mathrm{H}_{2} \mathrm{O}$ and $\mathrm{OH}$ and on their line profiles.

\section{A.3. Fits to $2.9 \mu \mathrm{m}$ Emission}

Figures $14-19$ show fits to CRIRES $2.9 \mu \mathrm{m}$ spectra made by using the $\mathrm{CO}$ line profiles as models for $\mathrm{H}_{2} \mathrm{O}$ and $\mathrm{OH}$ emission, as explained in Section 3.3. In addition to fitting the $2.9 \mu \mathrm{m}$ data with the $\mathrm{NC}$ or $\mathrm{BC}$ separately (in those disks that have two $\mathrm{CO}$ components), we also combine $\mathrm{NC}$ and $\mathrm{BC}$ profiles in different fractions, a technique that we call Spectral Principal Component Analysis (SPCA), as being based on principal spectral components given by $\mathrm{NC}$ and $\mathrm{BC}$ (Figure 15). In the double-component disks, we can therefore test whether $\mathrm{H}_{2} \mathrm{O}$ and $\mathrm{OH}$ are composed of one rather than two components, and which one dominates. To determine which model best represents the data among $\mathrm{NC}, \mathrm{BC}$, and their combination, we use the Akaike information criterion (Akaike 1974), defined as $\mathrm{AIC}=2 \times$ the number of free model parameters + the chisquare value. The criterion penalizes models with a larger number of free parameters, so that a simpler model is preferred unless the more complex model decreases the AIC number significantly (we adopt the criterion of a $\triangle \mathrm{AIC}>14$, corresponding to a $10^{4}$ higher probability of minimizing the information loss). In most cases, the chi-square value is enough to identify the best model between $\mathrm{BC}$ and $\mathrm{NC}$.
This procedure is generally robust against the photospheric correction performed on the $2.9 \mu \mathrm{m}$ velocity-resolved spectra obtained by CRIRES (Section 3.1), because the disk emission lines are typically broader than photospheric lines from the same molecules (Figures 2, 4, 12, 13) and because the high resolving power of CRIRES allows the separation of emission components with different velocities. In two disks (FN Tau, TW Hya), however, photospheric lines have similar width as the possibly detected $\mathrm{OH}$ emission lines, while $\mathrm{H}_{2} \mathrm{O}$ is undetected; their $\mathrm{OH}$ line fluxes and profiles are therefore highly uncertain (see Figure 18).

Overall, both non-detections and detections of weak emission advocate for the predominance of $\mathrm{BC}$ over $\mathrm{NC}$ in $\mathrm{H}_{2} \mathrm{O}$ and $\mathrm{OH}$ emission at $2.9 \mu \mathrm{m}$. In a few cases, spectra are best fit with a combination of BC and NC: specifically AS 205, DR Tau, and SCrA N (and only $\mathrm{OH}$ in CW Tau). These three disks are also the only ones where $\mathrm{NC}$ was detected in the $\mathrm{CO}$ $v=2-1$ lines, possibly due to the higher $\mathrm{S} / \mathrm{N}$ and dust veiling in these spectra compared to other spectra in the survey (Banzatti \& Pontoppidan 2015). Two other disks show tentative evidence for a weak NC, but its presence cannot be firmly established due to uncertainties from the photospheric correction: RU Lup and SCrA S. In all disks, the NC is about 2-10 times (or more) weaker in $\mathrm{H}_{2} \mathrm{O}$ and $\mathrm{OH}$ than in $\mathrm{CO}$, as compared to $\mathrm{BC}$. In a few cases only, the $\mathrm{OH}$ profile is better matched by $\mathrm{NC}$ rather than by BC (DF Tau, RNO 90, VW Cha). In single-component disks, $\mathrm{OH}$ is detected only in EC 82 and TW Cha.

\section{A.4. Luminosity and Accretion Trends}

Figure 20 shows the measured $\mathrm{H}_{2} \mathrm{O}$ and $\mathrm{OH}$ line fluxes plotted against the stellar + accretion luminosity, taken as a proxy for the irradiation and heating of inner disks. Only the 

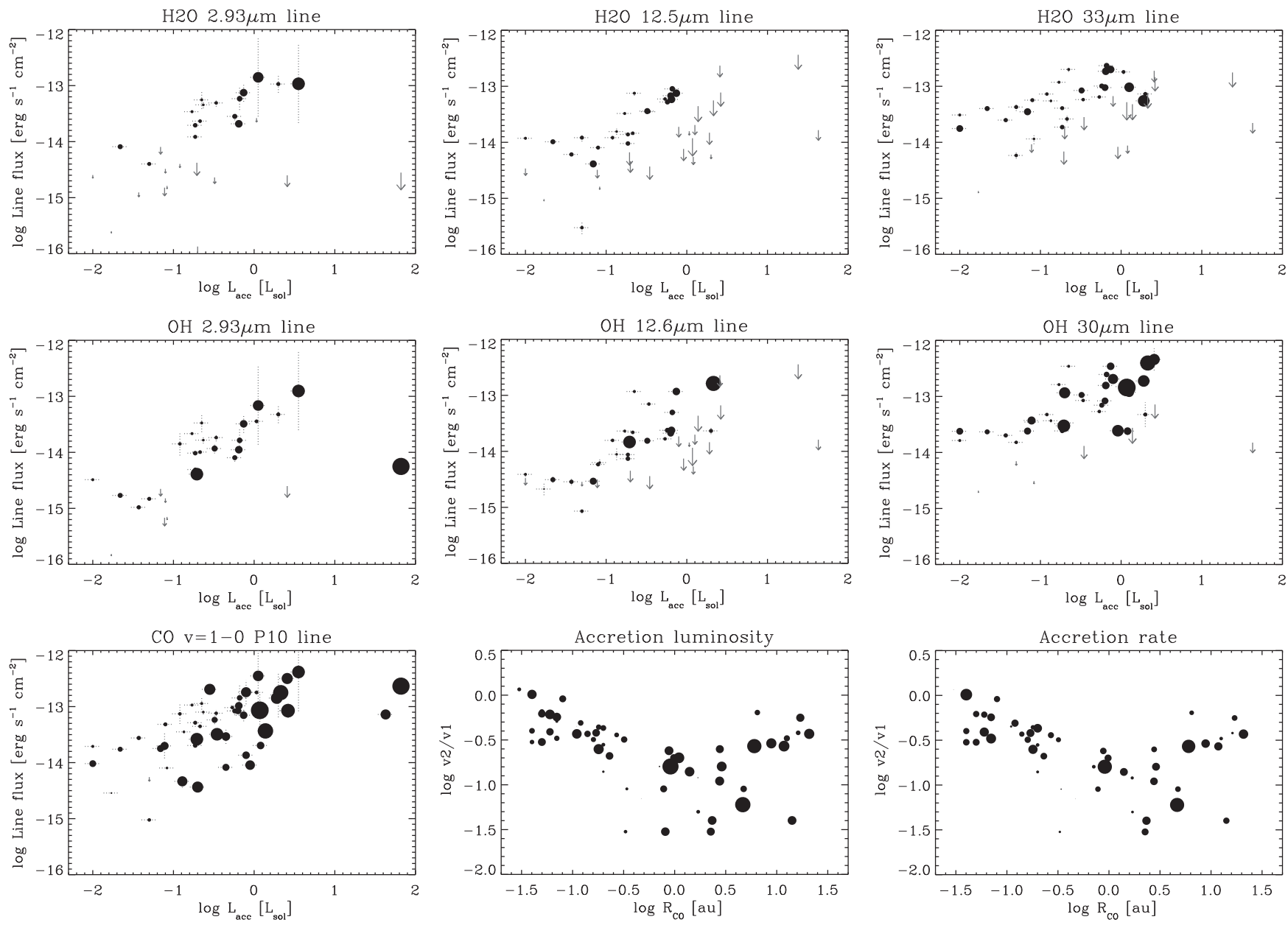

Figure 21. Dependence of measured line fluxes (normalized to $140 \mathrm{pc}$ ) on accretion luminosity. The symbol size is proportional to the stellar mass in panels showing line fluxes, while it is proportional to the logarithm of the accretion luminosity and rate in the last two panels, showing the TR diagram in the bottom right, as indicated.

$\mathrm{OH}$ line at $30 \mu \mathrm{m}$ shows a steady flux increase with increasing luminosity, consistent with an increasing area of the warm disk emitting region. Hotter $\mathrm{OH}$ lines show rather a turning point at a luminosity of $\sim 3 L_{\odot}$, beyond which line fluxes decrease (similar to that seen in the $\mathrm{H}_{2} \mathrm{O}$ at $33 \mu \mathrm{m}$ ). Figure 21 shows the measured line fluxes plotted against the accretion luminosity alone, to highlight how line fluxes overall increase with accretion luminosity. The last two plots in the bottom right of the figure show the T-R diagram of $\mathrm{CO}$ emission, highlighting how there is no global trend between the sequence of $\mathrm{CO}$ gaps and the accretion luminosity and rate. $\mathrm{OH}$ emission is more frequently detected than water emission in disks, and is tentatively detected at $30 \mu \mathrm{m}$ out to $R_{\mathrm{co}} / R_{\text {snow }} \sim 4$ (Figure 8) and up to larger luminosities (Figure 20), possibly supporting a still active photodissociation of trace amounts of $\mathrm{H}_{2} \mathrm{O}$ gas. $\mathrm{OH}$ formation through water photodissociation populates higher energy levels first (e.g., the $12.6 \mu \mathrm{m}$ line; see Carr \& Najita 2014), though, which are less often detected in disks that have a large $R_{\text {co. }}$. It is therefore unclear whether a cold $\mathrm{OH}$ reservoir traced at $30 \mu \mathrm{m}$ may be formed by photodissociation of $\mathrm{H}_{2} \mathrm{O}$ or by gas-phase chemistry, known to be efficient at temperatures of 100-300 K (e.g., van Dishoeck et al. 2013). Future observations of velocity-resolved $\mathrm{H}_{2} \mathrm{O}$ and $\mathrm{OH}$ lines at wavelengths longer then $2.9 \mu \mathrm{m}$ will be able to test if two reservoirs of $\mathrm{OH}$ exist: one due to UV photodissociation of water (with similar line profiles for $\mathrm{H}_{2} \mathrm{O}$ and $\mathrm{OH}$, as found at $2.9 \mu \mathrm{m}$ ), which dominates the hotter $\mathrm{OH}$ lines, and one due to $\mathrm{OH}$ formation in cold environments where water formation and/or release to the gas phase is less efficient (by finding larger line profiles for $\mathrm{H}_{2} \mathrm{O}$ and smaller line profiles for $\mathrm{OH}$ ).

\section{REFERENCES}

Akaike, H. 1974, ITAC, 19, 716

Akiyama, E., Hashimoto, J., Liu, H. B., et al. 2016, arXiv:1607.04708

Alencar, S. H. P., Bouvier, J., Walter, F. M., et al. 2012, A\&A, 541, A116

Alexander, R., Pascucci, I., Andrews, S., Armitage, P., \& Cieza, L. 2014,

Protostars and Planets VI, ed. H. Beuther et al. (Tucson, AZ: Univ. Arizona Press), 475

Alexander, R. D., Clarke, C. J., \& Pringle, J. E. 2006, MNRAS, 369, 229

Andrews, S. M., Wilner, D. J., Espaillat, C., et al. 2011, ApJ, 732, 42

Antonellini, S., Kamp, I., Lahuis, F., et al. 2016, A\&A, 585, A61

Antonellini, S., Kamp, I., Riviere-Marichalar, P., et al. 2015, A\&A, 582, A105

Aspin, C., Reipurth, B., Herczeg, G. J., \& Capak, P. 2010, ApJL, 719, L50

Bai, X.-N. 2016, ApJ, 821, 80

Banzatti, A., Meyer, M. R., Bruderer, S., et al. 2012, ApJ, 745, 90

Banzatti, A., Meyer, M. R., Manara, C. F., Pontoppidan, K. M., \& Testi, L. 2014, ApJ, 780, 26

Banzatti, A., \& Pontoppidan, K. M. 2015, ApJ, 809, 167

Banzatti, A., Pontoppidan, K. M., Bruderer, S., Muzerolle, J., \& Meyer, M. R. 2015, ApJL, 798, LL16

Basri, G., \& Batalha, C. 1990, ApJ, 363, 654

Bast, J. E., Brown, J. M., Herczeg, G. J., van Dishoeck, E. F., \& Pontoppidan, K. M. 2011, A\&A, 527, AA119

Bethell, T., \& Bergin, E. 2009, Sci, 326, 1675 
Blake, G. A., \& Boogert, A. C. A. 2004, ApJL, 606, L73

Blevins, S. M., Pontoppidan, K. M., Banzatti, A., et al. 2016, ApJ, 818, 22

Brittain, S. D., Najita, J. R., Carr, J. S., Ádámkovics, M., \& Reynolds, N. 2016, ApJ, 830, 112

Brittain, S. D., Rettig, T. W., Simon, T., et al. 2003, ApJ, 588, 535

Brittain, S. D., Simon, T., Najita, J. R., \& Rettig, T. W. 2007, ApJ, 659, 685

Brown, J. M., Pontoppidan, K. M., van Dishoeck, E. F., et al. 2013, ApJ, 770, 94 Bruderer, S. 2013, A\&A, 559, A46

Carmona, A., Thi, W. F., Kamp, I., et al. 2016, arXiv:1609.06708

Carr, J. S., \& Najita, J. R. 2011, ApJ, 733, 102

Carr, J. S., \& Najita, J. R. 2014, ApJ, 788, 66

Carr, J. S., Tokunaga, A. T., \& Najita, J. 2004, ApJ, 603, 213

Cutri, R. M., et al. 2012, yCat, 2311, 0

Cutri, R. M., et al. 2014, yCat, 2328, 0

Donati, J.-F., Bouvier, J., Walter, F. M., et al. 2011, MNRAS, 412, 2454

Dong, R., Zhu, Z., \& Whitney, B. 2015, ApJ, 809, 93

Espaillat, C., Ingleby, L., Hernández, J., et al. 2012, ApJ, 747, 103

Espaillat, C., Muzerolle, J., Najita, J., et al. 2014, in Protostars and Planets VI, ed. H. Beuther et al. (Tucson, AZ: Univ. Arizona Press), 497

Fairlamb, J. R., Oudmaijer, R. D., Mendigutía, I., Ilee, J. D., \& van den Ancker, M. E. 2015, MNRAS, 453, 976

Fedele, D., Bruderer, S., van Dishoeck, E. F., et al. 2012, A\&A, 544, L9

Fedele, D., Bruderer, S., van Dishoeck, E. F., et al. 2013, A\&A, 559, A77

Fedele, D., Pascucci, I., Brittain, S., et al. 2011, ApJ, 732, 106

Ferreira, J., Dougados, C., \& Cabrit, S. 2006, A\&A, 453, 785

Garufi, A., Quanz, S. P., Avenhaus, H., et al. 2013, A\&A, 560, A105

Gorti, U., \& Hollenbach, D. 2009, ApJ, 690, 1539

Gorti, U., Hollenbach, D., \& Dullemond, C. P. 2015, ApJ, 804, 29

Gray, R. O., Corbally, C. J., Garrison, R. F., et al. 2006, AJ, 132, 161

Haisch, K. E., Jr., Lada, E. A., \& Lada, C. J. 2001, ApJL, 553, L153

Hartigan, P., Hartmann, L., Kenyon, S., Hewett, R., \& Stauffer, J. 1989, ApJS, 70,899

Hartigan, P., Kenyon, S. J., Hartmann, L., et al. 1991, ApJ, 382, 617

Hein Bertelsen, R. P., Kamp, I., van der Plas, G., et al. 2016, A\&A, 590, A98

Herczeg, G. J., Brown, J. M., van Dishoeck, E. F., \& Pontoppidan, K. M. 2011, A\&A, 533, A112

Hoadley, K., France, K., Alexander, R. D., McJunkin, M., \& Schneider, P. C. 2015, ApJ, 812, 41

Hogerheijde, M. R., Bergin, E. A., Brinch, C., et al. 2011, Sci, 334, 338

Houck, J. R., Roellig, T. L., van Cleve, J., et al. 2004, ApJS, 154, 18

Ida, S., \& Lin, D. N. C. 2005, ApJ, 626, 1045

Ingleby, L., Calvet, N., Herczeg, G., et al. 2013, ApJ, 767, 112

Isella, A., Carpenter, J. M., \& Sargent, A. I. 2010, ApJ, 714, 1746

Johns-Krull, C. M., Valenti, J. A., \& Linsky, J. L. 2000, ApJ, 539, 815

Kaeufl, H.-U., Ballester, P., Biereichel, P., et al. 2004, Proc. SPIE, 5492, 1218

Kelly, B. C. 2007, ApJ, 665, 1489

Kennedy, G. M., \& Kenyon, S. J. 2008, ApJ, 673, 502-12

Kley, W., \& Nelson, R. P. 2012, ARA\&A, 50, 211

Koen, C., Kilkenny, D., van Wyk, F., \& Marang, F. 2010, MNRAS, 403, 1949

Lacy, J. H., Richter, M. J., Greathouse, T. K., Jaffe, D. T., \& Zhu, Q. 2002, PASP, 114, 153

Lagage, P. O., Pel, J. W., Authier, M., et al. 2004, Msngr, 117, 12

Lin, D. N. C., \& Papaloizou, J. 1986, ApJ, 309, 846

Maaskant, K. M., Honda, M., Waters, L. B. F. M., et al. 2013, A\&A, 555, A64

Manara, C. F., Fedele, D., Herczeg, G. J., \& Teixeira, P. S. 2016, A\&A, 585, A 136

Manara, C. F., Testi, L., Natta, A., et al. 2014, A\&A, 568, A18

Mandell, A. M., Bast, J., van Dishoeck, E. F., et al. 2012, ApJ, 747, 92

Mandell, A. M., Mumma, M. J., Blake, G. A., et al. 2008, ApJL, 681, L25

McLean, I. S., Becklin, E. E., Bendiksen, O., et al. 1998, Proc. SPIE, 3354, 566
Meeus, G., Montesinos, B., Mendigutía, I., et al. 2012, A\&A, 544, A78

Meijerink, R., Pontoppidan, K. M., Blake, G. A., Poelman, D. R., \& Dullemond, C. P. 2009, ApJ, 704, 1471

Menu, J., van Boekel, R., Henning, T., et al. 2015, A\&A, 581, A107

Min, M., Dullemond, C. P., Kama, M., \& Dominik, C. 2011, Icar, 212, 416

Morbidelli, A., Lunine, J. I., O’Brien, D. P., Raymond, S. N., \& Walsh, K. J. 2012, AREPS, 40, 251

Mulders, G. D., Ciesla, F. J., Min, M., \& Pascucci, I. 2015, ApJ, 807, 9

Najita, J. R., Ádámkovics, M., \& Glassgold, A. E. 2011, ApJ, 743, 147

Najita, J. R., Carr, J. S., Glassgold, A. E., \& Valenti, J. A. 2007a, Protostars and Planets V, 507

Najita, J. R., Carr, J. S., Strom, S. E., et al. 2010, ApJ, 712, 274

Notsu, S., Nomura, H., Ishimoto, D., et al. 2016, ApJ, 827, 113

Owen, J. E. 2016, PASA, 33, e005

Owen, J. E., Ercolano, B., Clarke, C. J., \& Alexander, R. D. 2010, MNRAS, 401, 1415

Pascucci, I., Herczeg, G., Carr, J. S., \& Bruderer, S. 2013, ApJ, 779, 178

Pontoppidan, K. M., Blake, G. A., \& Smette, A. 2011a, ApJ, 733, 84

Pontoppidan, K. M., Blake, G. A., van Dishoeck, E. F., et al. 2008, ApJ, 684, 1323

Pontoppidan, K. M., Salyk, C., Blake, G. A., et al. 2010a, ApJ, 720, 887

Pontoppidan, K. M., Salyk, C., Blake, G. A., \& Käufl, H. U. 2010b, ApJL, 722, L173

Pontoppidan, K. M., van Dishoeck, E., Blake, G. A., et al. 2011b, Msngr, 143, 32

Ribas, Á., Bouy, H., \& Merín, B. 2015, A\&A, 576, A52

Rigliaco, E., Pascucci, I., Duchene, G., et al. 2015, ApJ, 801, 31

Rivière Marichalar, P., Merín, B., Kamp, I., Eiroa, C., \& Montesinos, B. 2016, A\&A, 594, A59

Riviere-Marichalar, P., Bayo, A., Kamp, I., et al. 2015, A\&A, 575, A19

Riviere-Marichalar, P., Ménard, F., Thi, W. F., et al. 2012, A\&A, 538, L3

Rothman, L. S., Gordon, I. E., Babikov, Y., et al. 2013, JQSRT, 130, 4

Salyk, C., Blake, G. A., Boogert, A. C. A., \& Brown, J. M. 2011, ApJ, 743,112

Salyk, C., Herczeg, G. J., Brown, J. M., et al. 2013, ApJ, 769, 21

Salyk, C., Lacy, J. H., Richter, M. J., et al. 2015, ApJL, 810, L24

Salyk, C., Pontoppidan, K. M., Blake, G. A., et al. 2008, ApJL, 676, L49

Salyk, C., Pontoppidan, K. M., Blake, G. A., Najita, J. R., \& Carr, J. S. 2011, ApJ, 731, 130

Sicilia-Aguilar, A., Kóspál, Á., Setiawan, J., et al. 2012, A\&A, 544, A93

Simon, M. N., Pascucci, I., Edwards, S., et al. 2016, ApJ, 831, 169

Skrutskie, M. F., Dutkevitch, D., Strom, S. E., et al. 1990, AJ, 99, 1187

Strom, K. M., Strom, S. E., Edwards, S., Cabrit, S., \& Skrutskie, M. F. 1989, AJ, 97, 1451

Takeuchi, T., \& Artymowicz, P. 2001, ApJ, 557, 990

Thi, W. F., Kamp, I., Woitke, P., et al. 2013, A\&A, 551, A49

van der Marel, N., van Dishoeck, E. F., Bruderer, S., et al. 2016, A\&A, 585, A58

van der Plas, G., van den Ancker, M. E., Waters, L. B. F. M., \& Dominik, C 2015, A\&A, 574, A75

van Dishoeck, E. F., Herbst, E., \& Neufeld, D. A. 2013, ChRv, 113, 9043

Walsh, C., Nomura, H., \& van Dishoeck, E. 2015, A\&A, 582, A88

Werner, M. W., Roellig, T. L., Low, F. G., et al. 2004, ApJS, 154, 1

White, R. J., \& Ghez, A. M. 2001, ApJ, 556, 265

Woitke, P., Kamp, I., \& Thi, W.-F. 2009, A\&A, 501, 383

Woitke, P., Min, M., Pinte, C., et al. 2016, A\&A, 586, A103

Wright, E. L., Eisenhardt, P. R. M., Mainzer, A. K., et al. 2010, AJ, 140, 1868

Zhang, K., Pontoppidan, K. M., Salyk, C., \& Blake, G. A. 2013, ApJ, 766, 82

Zhu, Z., Nelson, R. P., Hartmann, L., Espaillat, C., \& Calvet, N. 2011, ApJ, 729, 47 